UM Libraries Depository

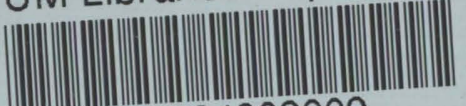

103334909009 

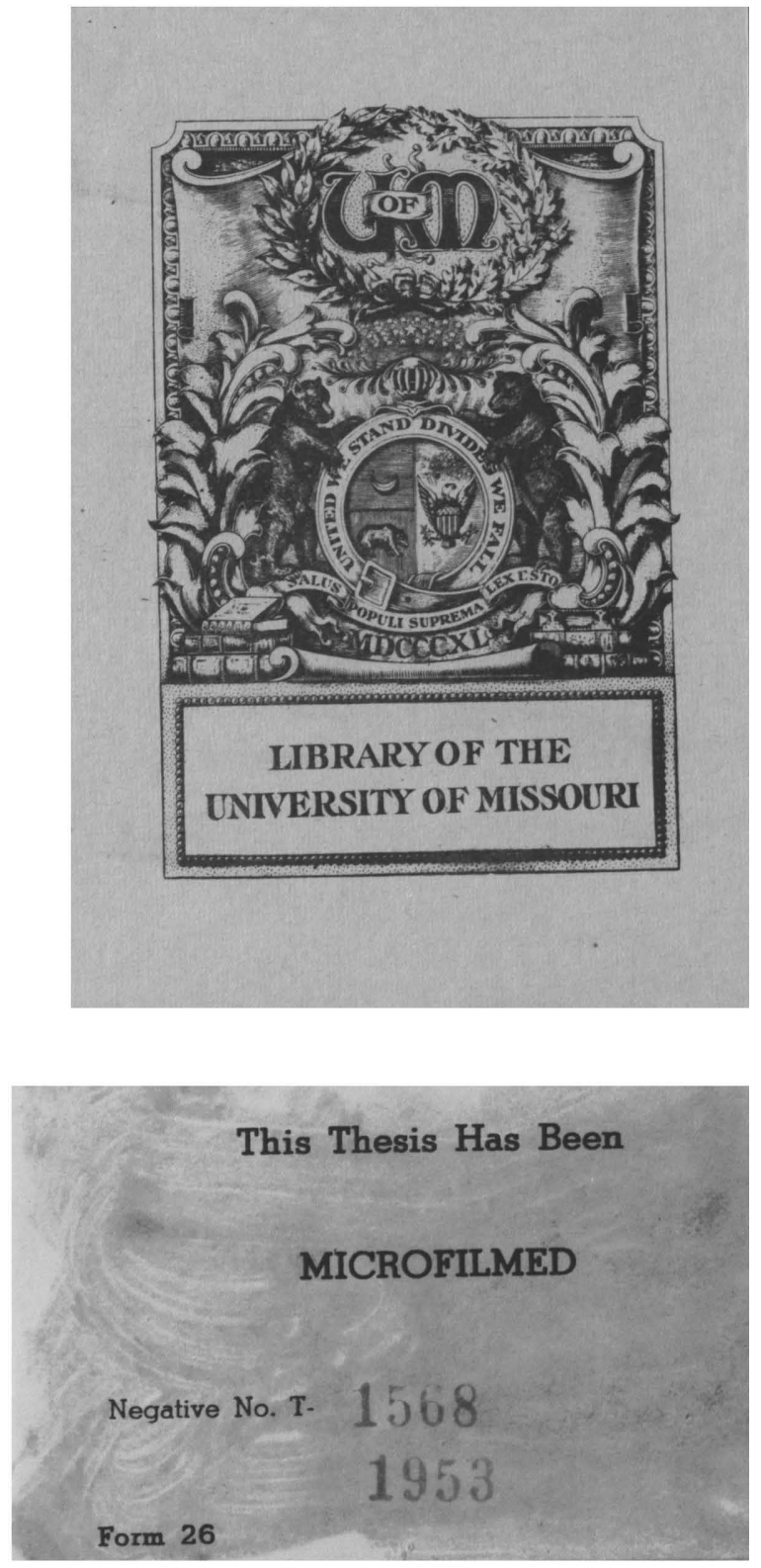




ON SOME CLASSES OF NON-ANALYTIC

FUNCTIONS OF A COMPLEX VARIABLE

by

EULA ADELI NE WEEKS, A.B.

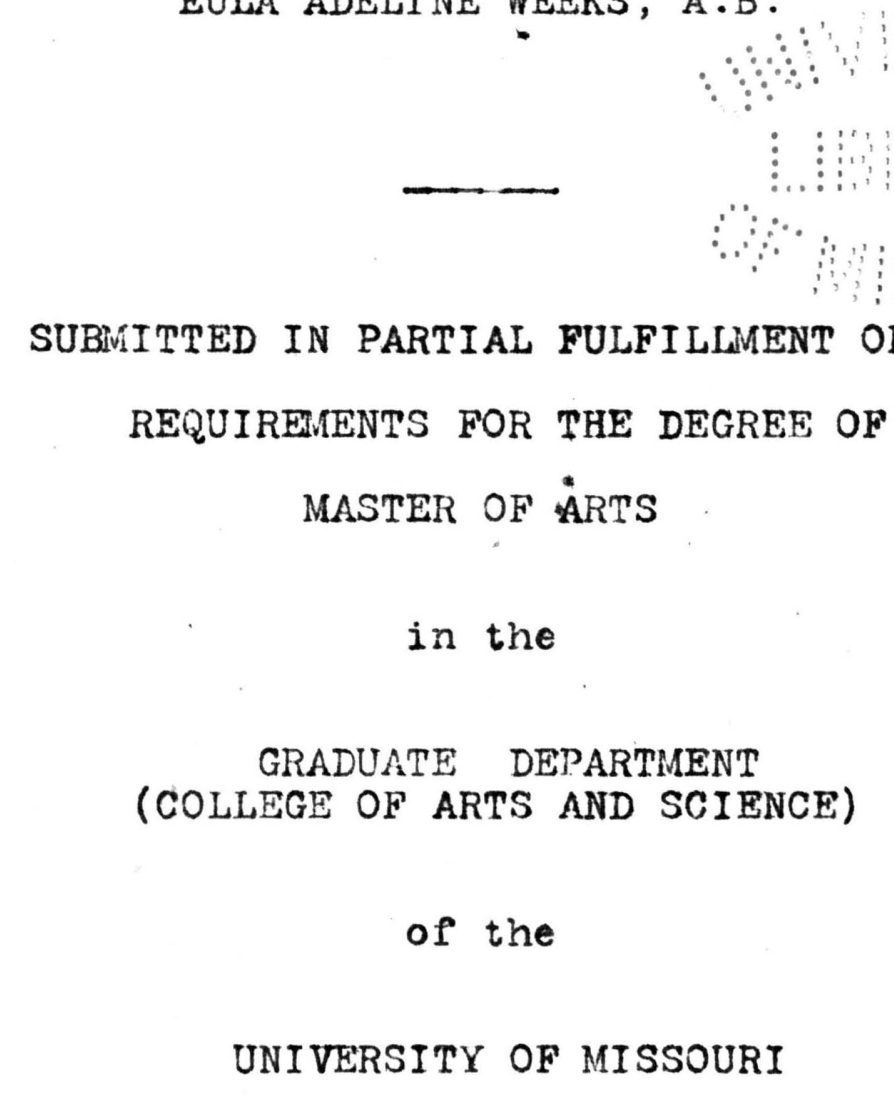

1909.

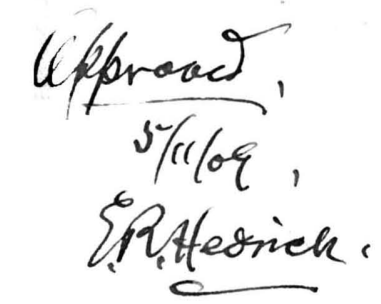



TABDE OF CONTHETS

Page

Introduction

1

$$
\text { Section } 1 .
$$

Analytic Functions: Fundamental Considerations

1. Geometrical Interpretation of Functions of

a Complex Varlable 10

2. A Unique Derirative in the Euclidean Plane 10

Section 21.

Other Classes of Functions: Fundamental Constderations

1. Geometrical Interpretation 13

2. Differentiation 13

3. Integration 18

4. Conformal Transformation of a Surface on Itself

Section $11 \lambda$.

Cauchy's Theorems, Taylor and Iaurent's Sertes and other Theorems

1. Continuous Functions 34

2. Regular Functions 35

3. Cauchy's Fundamental Formula 35

4. Cauchy's Integral Theorem 37 

5. Expressions for the Dexiratives 40

6. Tayzor'® Series 42

7. Iaurent's Series 43

8. Iiourizle'ø Theorem 49

9. The Singularities of Single-Valued Functions 50

10. Functions with No Fssential Singular Pointa 52

11. Coroliary 53

12. Iogarithmic Residues 54

13. Muntiple-Valued Functions: Branch Points 56

14. Ixample of Vany-Valued Functions 57

15. Region of Convergence 59

Section $2 V$.

1. Bxamples of Some of these Functions 61

2. Tnterpretation of this Work within the u, vaplane 65

Conclusion 67

Bibliography 1. 



\section{On Sore Classes of Non-Analytic Functions of a \\ Complex Variable}

\section{Introduction.}

In a study of the theory of analytic functions of a complex variable, one is at first impressed with the fact that postulating so little, one proves apparentiy so much. For instance, Professor Goursat in his proof of Cauchy's fundamental formula(i), assumes merely the existence of the first derivative of $f(z)$, and yet he arrives at the result that the number of derivatives of this function is unlimited - that is, that a derivative of any order not only exists, but is continuous. However, one must bear in mind that this class of function, known as analytic, is a very limited and special class; for the analytic functions $w=f(z)$, where $w=u+v i$ and $z=x+y 1$, are limited to the solutions of the CauchyRiemann equations, $\frac{\partial u}{\partial x}=\frac{\partial v}{\partial y}, \frac{\partial v}{\partial x}=-\frac{\partial u}{\partial y}$.

This fact, namely, that the analytic functions are a very limited and special class, with the additional fact that there seems to be no reason a priori why many of the theorems concerning analytic functions cannot be extended to analogous theorems for non-analytic functions, lead us to seek to define other classes of functions of a complex var-

(1) E. Goursat: Cours d'Analyse Mathematique. Vol. II.,p.82. 

iable and to attempt to find for the classes, thus defined, theorems analogous to those for analytic functions.

Before we attack the problem of defining these new classes of functions, let us first see what meaning has been attached to the term, function of a compiex variable, and what has been the attitude in regard to the comprehensiveness of the theory of analytic functions.

Since the definitions of a function of a complex variable have been extensions of the definitions of functions of reat variables, it is interesting to note the process by which we have reached the modern conception of functions of a real variable. To the older mathematicians, a function of $\underline{x}$ usually meant nothing more than a power of $\underline{x}$; and certainly their broadest defintion of function of $\underline{x}$ would have included only explicit functions of $x$. Euler extended the definition to include implicit functions of $\underline{x}$. And through a consideration of trigonometric series, Fourier was lead to a definition of function which admitted different analytic expressions in different intervals. However, it was Dirichlet, who gave us the broad definition which we have today, namely, $y$ is a single valued function of the variable $x$, in the continuous interval $(a, b)$, when a definite value of $y$ corresponds to esch value of $x$, such that $a \leq \underline{x} \leq b$, no matter in what form this correspondence is satisfied.(1)

(i) Hobson: Functions of a Real Variable. p. 216. Pringsheim: Encyklopadie der mathematischer Wissenschaften, II. A I, pp. $3-8$. 

Thus we see that the broad definition of a function of a real variable which we have today, was the culmination of a slow process.

In contrast with this, we find among the earliest writers on the theory of functions of a complex variable a broad definition of functions of a compiex variable. However, we do find, even among some of the modern writers on the theory of functions of a complex variable, a narrow definition of function( 1$)$ And it seems that in some cases, at least, this narrow definition of function is due rather to lack of breadth of view of the function theory than to a conscious effort to narrow the definition so that it will include only analytic functions.

We have said that the analytic functions of a complex variable are merely those functions which are solutions of the Cauchy-Riemann equations. But it is scarcely necessary to state that this limitation of the theory of functions of a complex variable did not come about by the arbitrary decision of some one person, or persons, to study onIy the functions which satisfy these particular differential equations. When men first attempted to handle the simple functions of a complex variable which they first encountered, it was but natural to follow along the lines of the theory of functions of a real variable and to dernand the existence

\section{(i) Some of these definitions of a complex variable will be quoted later.}



of a derivative in the Euclidean plane. It was this demand, and not any arbitrary choice, which lead to the Cauchy-Rieman(i) equations. From the present development of the function theory, it is easy to see why they were lead to the Cauchy-Riemann equations; for the simplest functions are, in general, developable in the Cauchy-Taylor series, and all functions which are developable in this series are solutions of these equations.

Strictiy speaking, no one person can be said to have originated the theory of functions of a complex variable, and yet Cauchy, who first made an attempt to give both a rigorous and a general treatment of functions of a complex variable, may be regarded as its true founder.

Cauchy's memoir - Sur les fonctions de variables imaginaires $^{(i i)}$ - not onty is one of the earliest contributions to the theory of functions of a complex variable, but it also contains the very essence of the spirit of modern analysis; for this reason, it is apparent that no better introduction to the theory of functions of a complex variable can be given than this mathematical classic:-

"La théorie des fonctions de variables imaginaires présente des questions délicates qu'il importait de résoudre, et qui ont souvent embarrassé les géomètres. Mais toute difficulté disparaîtra, si, en se laissant guider par 1 'analogie, on étend aux fonctions de variables imaginaires

(i) Riemann's demand is that the integral be independent of the path.

(ii) Comptes Rendus: $1851,2^{e}$ sem. p. 160. 

"les definitions generalement adoptees pour les fonctions de variables réeles. On arrive ainsi à des conciusions, singulieres au premier abord, et néanmoins très-légitjmes, que j'indiquerai en peu de mots.

"Deux variables réles sont dites fonctions $I^{\text {'une }}$ de $I^{\prime}$ autre, lorsqu'elies varient simultanement de telie sot te que 1 a valeur de 1 'une déternine la valeur de l'autre. Si les deux variables sont censées représenter les abscisses de deux points assujettis a se mouvoir sur une même droite, la position de 1 'un des points mobiles déterminera la position de l'autre, et reciproquement.

"Ajoutons que le rapport differentiel de deux variables réeles est une quantité géneralement deterninée, et qui nóanmoins peut cesser de 1 'être pour certaines valeurs particulieres des variables. Ainsi, par exemple, le rapport différentiel de $y$ a $x$ deviendra indéterminé pour $\underline{x}=0$, si l'on suppose

$$
y=x \sin \frac{1}{x} \text {. }
$$

"Concevons maintenant que, $x, y$ trant des variables reelles et indépendantes $\frac{1}{Z}$ 'une de. I'autre, on pose

$$
\bar{z}=x+y^{i}
$$

$i$ etant une racine carree de -1 . $z$ sera ce qu'on nomme une variable Imaginaire. Soit

$$
u=V+W_{i}
$$

une autre variable imaginaire, v et $\mathbf{w}$ étant réels. Si, comme on doit natureldement de faire, on étend aux variables imaginaires les définitions adoptées dans le cas ou les variables sont réelles, $u$ devra être censé fonction de $\underline{z}$, lorsque la valeur de $z$ déterminera la valeur de $\underline{u}$. Or, $1 \bar{I}$, suffit pour cela que $\underline{v}$ et soient des fonctions déterminés de $x, y$. Alors aussí, en considérant les variabies réeles $\underline{x}$ et $y$ renfermees dan $\underline{z}$, ou les variables réelles $\underline{v}$ et $\underline{w}$ renfermés dans $u$, commepropres à representer les coordōnnées rectilignes et rectangulalres, d un point mobile $z$ ou $U$, on verra la position du point mobile $\mathrm{Z}$ déterminer toujours la position du point mobile U.

"Si d'ailieurs on nomme $r$ le rayon vecteur mené de 1 'ortgine des coordonnees au point mobile $Z$, et $p$ l'angle polaire formé par ce rayon vecteur avec l'axe des $x, 1 \in s$ coordonnées polaires $\underline{r}$ et $\underline{p}$, liées a $\underline{x}, \underline{y}$ par les equations

et a z par la formule

$$
x=r \cos p, \quad y=r \sin p
$$

$$
z=r e^{p i}
$$

seront ce qu'on nomme le module et l'argument de la variable imaginaire $z$.

"Ces définitions étant adoptés, et y étant une fonction quelconque de la variable imaginaire' $z$, le rapport differentiel de $\underline{u} a \underline{z}$ dépendra, en general non-seulement des variables rélies $\bar{x}$ et ou, ce qui revient au meme, de la position attribuee au point mobile $z$, mais encore du rapport differen- 

tiel de $y$ a $x$, ou, en d'autres termes, de la direction de tangente a la courbe que decrira le point mobile, lorsq'on fera varier z. Ainsi, par exemple, comme on aura

$$
d z=d x
$$

si le point mobile se meut paralielement à 1 'àxe des $x$, et

$$
d z=i d y_{\text {. }}
$$

si te point mobile se meut parallelement à $l$ 'axe des $y$, le rapport différentiel de $\underline{u}$ a $\underline{z}$ sera, dans la première hypothese,

$$
D_{x} v+i D_{x} w
$$

et, dans 1 a seconde hypothese,

$$
\frac{D_{y} v+i D_{y} w}{i}=D_{y} w-i D_{y} v \text {. }
$$

Ajoutons que, si ces deux valeurs particulières du rapport différentiel de u à $z$ sont égales entre elles, ce rapport deviendra independant de la direction sulvie par le point mobile, et se réduira simplement a une fonction des deux variables $x, y$.

"Dans ce cas particulier, on aura

par conséquent,

$$
D_{x} v=D_{y} w, \quad D_{y} v=-D_{x} w \text {; }
$$

et

$$
D_{x}^{2} v+D_{y}^{2} v=0, \quad D_{x}^{2} W+D_{y}^{2} W=0_{j}
$$

$$
D_{x}^{2} u+D_{y}^{2} u=0 \text {. }
$$

Donc alors la fonction $\underline{u}$ de $z$ sera en même temps une fonction de $x$, y qui vérifiera uñe equation aux dérivees partielies du secona ordre, et représentera une integrale de cette equation.

"C'est ce qui arriverg ordinairement, si les, variables imaginaires uet $z$ sont liees entre elles par 1 equation qu'on obtient en égalant á zéro une fonction toujours continue de ces deux variables.

Les principes que je viens dexposer confirment ce que j'ai dit ailleurs sur la nécessité de mentionner la dérivée d'une fonction de $\underline{z}$, dans le theoreme qui indique les conditions sous lesquelies cetter fonction peut être développee en une série oráonnée suivant les puissances ascendantes de z. C'est, au reste, ce que j'expliquerai plus en détail dans un autre article, ou je déduirai des principes dont il $s^{\prime a g i t}$ les propriétés diverses des fonctions d'une variable imaginaire et de leurs intégrales définies."

It seems that the spirit of modern analysis - by which is meant a certain open-mindedness and breadth of vier - 

a realization not only of the present limitations of the function theory, but also of the possibility of its extension - is shown in Cauchy's broad definition of function of a complex variable, and in his realization of the very limited nature of the class of functions with which he is dealing.

As we previously mentioned, this broad definition has not been accepted by all the later writers on function theory. Harnack applied the term function of a complex variable to $\boldsymbol{w}=f(z)$, when $w$ is produced by means of any finite number of arithmetical operations on $\underline{z}^{(i)}$ Professor For-

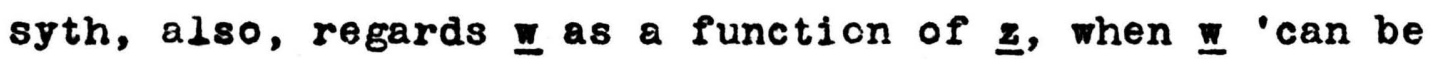
constructed by definite operations $\left(j \frac{j}{b}\right) z$ regarded as an irresoluble magnitude.'(iii) Durege, who certainly is willing in general to give to the term function its broadest interpretation, feels that when we pass to the domain of complex numbers we must limit our term function; hence, he applies the term function of a complex variable only to those func-

(i) Harnack: Introduction to Calculus. p. 128. (ii) A conception of function which is not very clear, since one cannot be certain just what Prof. Forsyth means by definite operations. It might, also, be noted that Harnack's definition would inc ude infinite series, and whereas, a uniformly convergent series of "anaiytic" functions is an "analytic" function; it is not in general true that a convergent series of "analytic" functions is an "analytic" function:- hence, Harnack's definition, strictiy accepted, would realiy include more than he intends, i.e., more than the class of "analytic" functions, and his theorems wo ld not strictly follow. The same conctusions apply to Prof. Forsyth's definition, if it be refined sufficlently so as to express a perfectly definite class of operations.

(iii) Forsyth: Theory of Functions. p.7. 

tions where $\underline{x}$ and $y$ occur in the combination $x+y i$. He, too, then demands that in a function of $\underline{z}, \underline{z}$ appear as an Irresoluble quantity.(i) However, he does recognize that this demand gives him a very limited class of function, and those functions which he excludes from his considerations he cal1s complex functions, not functions of a complex variable. Professor osgood does not deviate from Cauchy's statement when he defines function as follows Eine Funktion $f(z)$ einer complexen Verunderlichen entsteht dadurch, dass man jedem Punkte $\underline{z}$ eines Bereiches $T$ der Komplexen Zahlenebene eine Zahl

$$
w=u+v i=f(z)
$$

nach einem bestimmeten Gesetze zuoranet"(ii) professor Osgood certainiy realizes the existence of functions of a complex rariable other than the anaigtic functions, and he proves theorems about functions on which he has not placed the restriction that they be analytic.

Whether we accept the broad definition of a function of a complex variable given by Cauchy, or with Durege consider all non-analytic functions, not as functions of a complex variable, but as complex functions; we must realize that besides the analytic functions of a complex variable, there is an infinity of classes of functions, each class of which is coextensive in totality with the anatjtic functions

(1) Durege: Eiements of Theory of Functions. p.27.

(i1) 0sgood: Lehrbuch der Funkt1onen Theorie. p. 180. 

and about which the ordinary function theory postulates nothing, proves nothing.

In speaking of the development of the theory of functions of a complex variabie, it was said that those simple functions of a complex variable which were first encountered were anaiytic; but, while this is ture, it must not be inferred that analytic functions inciude ald those which are most simple and most useful, and non-anaLytic functions oniy those which are difficult and useless. The division into anasytic and non-ana+ytic functions is made on the basis neither of simplicity nor of usefulness. And what Professor Osgood says of anatytic and non-ana $\perp y-$ tic functions of a real variabie applies equaldy well to analytic and non-analytic functions of a complex variable:"Demgemass entspricht die Einteilung der Funktionen in anaLytische und nicht-analytische nicht der Natur der Sache."(i) Therefore in an extension of the theory of functions of a complex rariable to include non-anatytic functions, we are not necessaridy considering functions which are tess simple or less useful.

(i) Osgood: op. cit., p. 95 . 

Section I.

1. Geometrical Interpretation of Functions of a complex Variable.

In the development of the function theory, probably nothing has proved to be more helpful than the visualization of the dependence of the one variable upon the other.

The geometrical interpretation of functions of a complex variable offers far greater difficulty than that of functions of a real variable, because given $w=f(z)$, where $w=u+v i$ and $z=x+y 1$, we have four variables, and hence a geometrical representation of functions of a complex variable, analogous to that for functions of a real variable, would necessitate four dimensions.

There have been several suggestions(i) offered as to possible geometrical interpretations of functions of a complex variable. The one which has been most frequently used is to consider $w=f(z)$ as a transformation of the $z-$ plane upon the w. It can be shown that all the theory of analytic functions of a complex variable can be interpreted as a conformal transformation of a plane upon itself.

2. A unique derivative in the Euclidean plane.

Let $w=f(z)$ be a single-valued continuous function of $z$, where

$$
\begin{aligned}
& z=x+y i, \\
& w=u+v i .
\end{aligned}
$$

Let us consider this function $w=f(z)$ as defining a trans-

(1) Whittaker: Modern Anajysis. p. 41. 

formation of $\underline{z}$ upon the w plane. To every point of $\underline{z}$ in the $z$-plane will correspond a point in the w-plane.

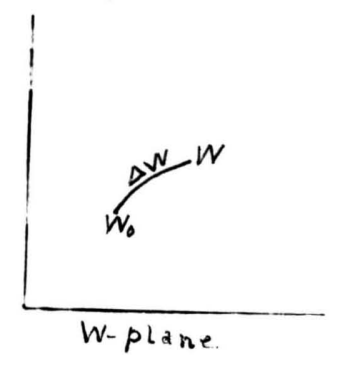

Fig. 1.

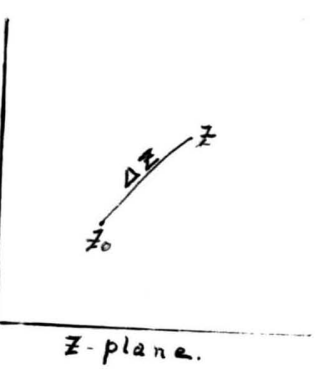

Take any' two points $z^{\prime}$ and $z_{0}$ in the $z$-plane, and there will correspond two points $w$ and $w_{0}$ in the $w-p l a n e$. Let the point $z$ approach the point $z_{0}$ along any path whatever, then the point w will approach wo along the w-plane. The demand for the existence of a unique derivative is that the limit of the ratio $\frac{\Delta}{\Delta} \frac{W}{Z}$ be independent of the path along which $z$ approaches $z_{0}$.

(1) $\frac{d w}{d z}=\lim _{\Delta z=0} \frac{\Delta w}{\Delta z}=\lim _{\substack{\Delta x=0 \\ \Delta y=0}} \frac{\Delta U+\Delta v_{i}}{\Delta x+\Delta y i}$,

multiplying through by

this becomes

$$
\frac{\Delta x-\Delta y i}{\Delta x-\Delta y i}
$$

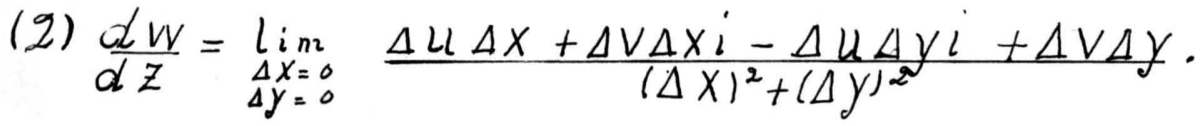

Dividing both numerator and denominator by $(\Delta X)^{2}$, we have,

(3) $\frac{d w}{d z}=\lim _{\substack{\Delta x=0 \\ \Delta y=0}} \frac{\frac{\Delta u}{\Delta x}+\frac{\Delta V}{\Delta x} i-\frac{\Delta u}{\Delta y}\left(\frac{\Delta y}{\Delta x}\right)^{2} i+\frac{\Delta V}{\Delta y}\left(\frac{\Delta y}{\Delta x}\right)^{2}}{1+\left(\frac{\Delta y}{\Delta} \frac{y}{x}\right)^{2}}$. 

Passing to the limit, we have

(4) $\frac{d w}{d z}=\frac{\frac{\partial u}{\partial x}+\frac{\partial v}{\partial x} i-\frac{\partial u}{\partial y}\left(\frac{d y}{d x}\right)^{2} i+\frac{\partial v}{\partial y}\left(\frac{d y}{d x}\right)^{2}}{1+\left(\frac{d y}{d x}\right)^{2}}$.

Now if the derivative $\frac{d w}{d z}$ is to be unique, the qbove ratio must be independent of $\frac{d y}{d x}$, hence if this condition be fulfilled, we have

$$
\frac{\frac{\partial u}{\partial x}+\frac{\partial v}{\partial x} i}{1}=\frac{-\frac{\partial u}{\partial y} i+\frac{\partial v}{\partial y}}{1} \text {. }
$$

Equating real and imaginary parts, we have

(6) $\quad \frac{\partial u}{\partial x}=\frac{\partial v}{\partial y}, \frac{\partial v}{\partial x}=-\frac{\partial u}{\partial y}$,

which are the Cauchy-Riemann equations. If we make the demand that the integral from $a$ to $\underline{b}$ of $f(z)$ be independent of the path, we are lead to the same differential equations. These Cauchy-Riemann equations glve us not on 1 y a conformal, but also an isogonal transformation of the plane upon itself. (i)

(i) For these and further proof of theorems about analytic functions $8 \theta e$ any standard work on Function Theory; for example, Goursat: Cours d'Anadyse. Vol. II. 



\section{Section II.}

Other Classes of Functions - Fundamental Considerations

1. Geometrical Representation.

In order to obtain other classes of functions of a complex variable which are readily studied, we shall accept the broad definition of a function, that is, that is a function of $\underline{\underline{r}})^{(1)}$ if, whenever $\underline{\underline{r}}$ is given, $\underline{\underline{t}}$ is defined; but we shall make different demands from those make upon the analytic functions. One concelvable method of placing different restrictions upon the functions is this Consider the function $w=w(r)$ as a transformation of a surface(ii) upon itself, and then work along lines analagous to those followed in the theory of analytic functions.

2. Differentiation.

Let the surface $\sum$ be defined by the equations,

$$
\begin{aligned}
& x=f(p, q), \\
& y=\phi(p, q), \\
& z=\psi(p, q),
\end{aligned}
$$

and also let us choose the function

$$
w=v(r)=u+v i \text {. }
$$

(i) For the sake of notation, since we use $z$ in the equations which define the surface, we shall use $w=w(r)$, where $w=u+v i$, and $r=p+q i$, instead of $w=f(z)$.

(ii) Every essentialiy different class of surfaces defines a different class of functions. The function of each class are as "numerous" in the sense of the theory of assemblages as the analytic functions. 

which gives rise to the equations

$$
\begin{aligned}
& u=u(p, q) \\
& V=V(p, q),
\end{aligned}
$$

in such a way that

$$
d u u^{2}+d v^{2}=\lambda\left(E d p^{2}+2 F d p d q+G d q^{2}\right)
$$

where $\lambda$ is a function of $p$ and $\phi$ alone, (i) and $E, F$, and $G$ mean the following:

(10)

$$
\begin{aligned}
& E=\left(\frac{\partial x}{\partial p}\right)^{2}+\left(\frac{\partial y}{\partial p}\right)^{2}+\left(\frac{\partial z}{\partial p}\right)^{2} \\
& F=\frac{\partial x}{\partial p} \cdot \frac{\partial x}{\partial q}+\frac{\partial y}{\partial p} \cdot \frac{\partial y}{\partial q}+\frac{\partial z}{\partial p} \partial y \\
& G=\left(\frac{\partial x}{\partial q}\right)^{2}+\left(\frac{\partial y}{\partial q}\right)^{2}+\left(\frac{\partial z}{\partial q}\right)^{2}
\end{aligned}
$$

Let

$$
W^{\prime}=\phi(r)=u^{\prime}+v^{\prime} i
$$

be arbitrary function of $\underline{\underline{z}}$; since $v^{\prime}=u^{\prime}+v^{\prime \prime}$ gives rise to the equations

$$
\begin{aligned}
& u^{\prime}=N(u, v)=N(u(p, q), v(p, q))=A(p, q) \\
& v^{\prime}=N(u, v)=N(u(p, q), v(p, q))=B(p, q), \\
& w^{\prime}=u u^{\prime}+v i=A(p, q)+B(p, q) i, \\
& w^{\prime}=M(u, v)+N(u, v) i=F(v v) .
\end{aligned}
$$

(1) This is the condition that the function $w=u+v i d \theta-$ fine a conformal transformation of the surface $\sum$ upon the u,v-plane Cf. E.Plcard: Traite d'Analyse. Vol. I. p. 477. (ii) For the derivation of these equations and the expression for the square of the linear element see E. Picard: op. cit. vol. I. p. 445, or Darboux: Lecons sur la general Theorie surface. vol. I., p. 74. 

Let us consider $w^{\prime}=\phi(r)=u^{\prime}+v^{\prime} i$ as a transformation of the surface $\sum$ upon itself, and let us assume that the functions $u, v, u^{\prime}, v^{\prime}, x, y$, , and $z$ admit first partial derivatives with respect to $p$ and $q$.

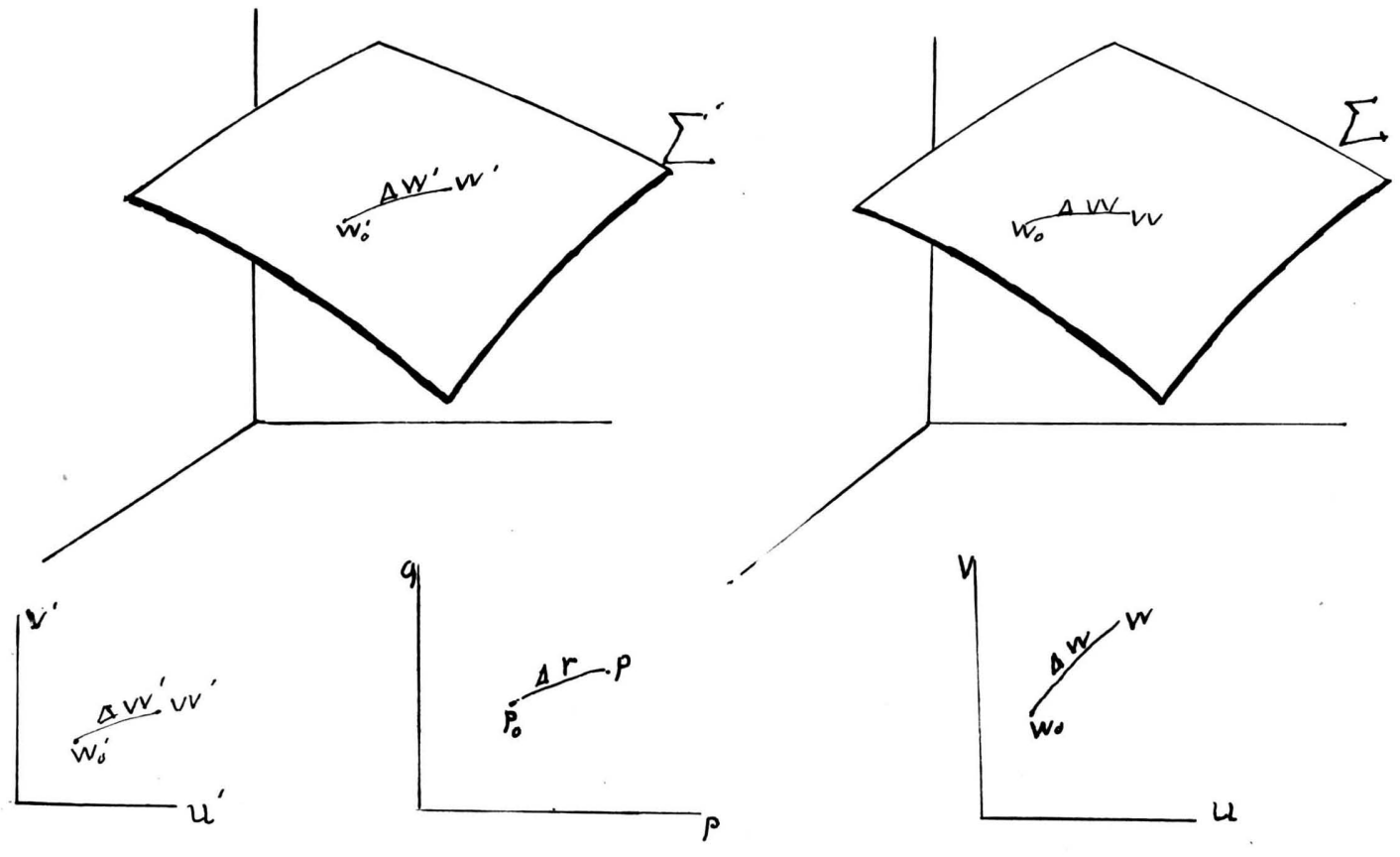

Fig. II.

If we take any two points $p$ and $p_{0}$ in the $p, q-$ plane, and any path joining these points, then we have two corresponding points $w$ and $v_{0}$ with a corresponding path joining them, in the $u, v$-plane; and in the $u^{\prime}, v^{\prime}$-plane corresponding points $w^{\prime}, w_{0}^{\prime}$, with a corresponding path joining them. On the surface $\sum$ there will be two sets of points $W, W_{0}$, and $W^{\prime}, v_{o}^{j}$ corresponding to $p$, and $p_{0}$ connected by paths corresponding to the path pop. (We have made two representations of the surface $\Sigma$, in order that the geometricaldmeaningof the work following may be more easily seen. However, we may think of the second 

representation as being a transparent surface placed directiy over the first representation of $\sum$. $W$ and $v v^{\prime}$ merely define a different parameter representation of the surface.)

The most natural demand to make upon this function $w^{\prime}=F(w)$ is that it have a unique, definite derivative. The demand for a unique, definite derivative of $V V$ 'with respect to $V V$ is the same as the demand that the limit of the ratio $\frac{\Delta w^{\prime}}{\Delta v V}$, as $v v$ approaches $w_{0}$, be independent of the path of approach, that is, be independent of the manner in which $P$ approached $p_{0}$.

In this case, as in the case of anatytic functions, we shall consider the vector differences $\Delta v v$ and $\Delta v v^{\prime}$. We have

$$
\frac{\Delta W^{\prime}}{\Delta W}=\frac{\left(u u^{\prime}-2 u_{0}^{\prime}\right)+\left(v^{\prime}-v_{0}^{\prime}\right) i}{\left(u-2 L_{0}\right)+\left(v-v_{0}\right) i}=\frac{A_{p} \Delta p+A_{q} \Delta q+\left(\beta_{p} \Delta p+\beta_{q} \Delta q\right) i}{\left.\left.u_{p} \Delta p+u_{q} \Delta q+v_{p} \Delta p+v_{q} \Delta\right)^{\prime}\right)}
$$

dividing through by $4 p$ and passing to the limit, the above equation becomes

(11) $\frac{d v_{v}{ }^{\prime}}{d v v}=\lim _{\substack{\Delta p \rightarrow 0 \\ \Delta q=0}} \frac{\Delta w^{\prime}}{\Delta w}=\frac{A_{p}+A q d q}{u_{p}+\left(\beta_{p}+\beta_{q} \frac{d q}{d p}\right) i}$

In order that $\frac{d w^{\prime}}{d v}$ be independent of the path, the above ratio must be independent of $\frac{d g}{d p}$, hence

$$
\frac{A p+i B_{p}}{A_{p}+i v p}=\frac{A_{q}+i B_{q}}{u_{q}+i v q} \text {. }
$$

which reduces to the two equations,

$$
\begin{aligned}
& u_{p} A_{q}-v_{p} B_{q}=u q A_{p}-v_{q} B_{p} . \\
& u_{p} B_{q}+v_{p} A_{q}=u q B_{p}+v_{q} A_{p} .
\end{aligned}
$$



These equations for the classes of functions which we are to consider are the analogons of the Cauchy-Riemann equations for the analytic functions. These equations may be written in the form

(14)

$$
\left|\begin{array}{cc}
u_{p} & u_{q} \\
A_{p} & A_{q}
\end{array}\right|=\left|\begin{array}{ll}
v_{p} & v_{q} \\
B_{p} & B_{q}
\end{array}\right|
$$

$$
\left|\begin{array}{ll}
u_{p} & u_{q} \\
B_{p} & B_{q}
\end{array}\right|=-\left|\begin{array}{ll}
v_{p} & v_{q} \\
A_{p} & A_{q}
\end{array}\right|
$$

or, if we make use of the equations

$$
\begin{aligned}
& \lambda E=u_{p}^{2}+v_{p}^{2} \\
& \lambda F=u_{p} u_{q}+v_{p} v_{q} \\
& \lambda G=u_{q}^{2}+v_{q}^{2}
\end{aligned}
$$

and solve for $A_{p}$ and $A_{q}$, we have

(15)

$$
\frac{\partial A}{\partial p}=\frac{E \frac{\partial B}{\partial q}-F \frac{\partial B}{\partial p}}{\sqrt{E G-F^{2}}}
$$

$$
\frac{\partial A}{\partial q}=\frac{F \frac{\partial B}{\partial Q}-G \frac{\partial B}{\partial P}}{\sqrt{E G-F^{2}}}
$$

which are known as the Beltrami equations. It has been pointed out by Professor Picard that the equations (15) may be regarded as a generalization of the equations (6), and that the equation of the second order derived from them

$$
\frac{\partial}{\partial p}\left(\frac{G \frac{\partial B}{\partial P}-F \frac{\partial B}{\partial Q}}{\sqrt{E G-F^{2}}}\right)+\frac{\partial}{\partial q}\left(\frac{E \frac{\partial B}{\partial q}-F \frac{\partial B}{\partial P}}{\sqrt{E G-F^{2}}}\right)=0 .
$$

(i) These come directly from equation(9). 

is on the surface $\sum$ the analogon of the Laplace equation on the plane! (i)

3. Integration.

We now pass to a consideration of integration for this class of functions.

Let us consider, as in the case of differentiation, that we have a surface $\sum$ defined by the equations,

$$
\begin{aligned}
& x=f(p, q) \\
& y=\varphi(p, q) \\
& z=\psi(p, q),
\end{aligned}
$$

and also let us choose $w=u+v i=u(q, q)+v(p, q) i$ in such a way that

$$
d u^{2}+d v^{2}=\lambda\left(E d p^{2}+2 F d p d q+G d_{q}^{2}\right)
$$

where $\lambda$ is a function of $p$ and $q$ (ii)

Let us take

$$
\left.w^{\prime}=u^{\prime}+v^{\prime} i=F(v)=A(p, q)+B(p, q)\right)_{i}^{\prime}=\phi(r) \text {. }
$$
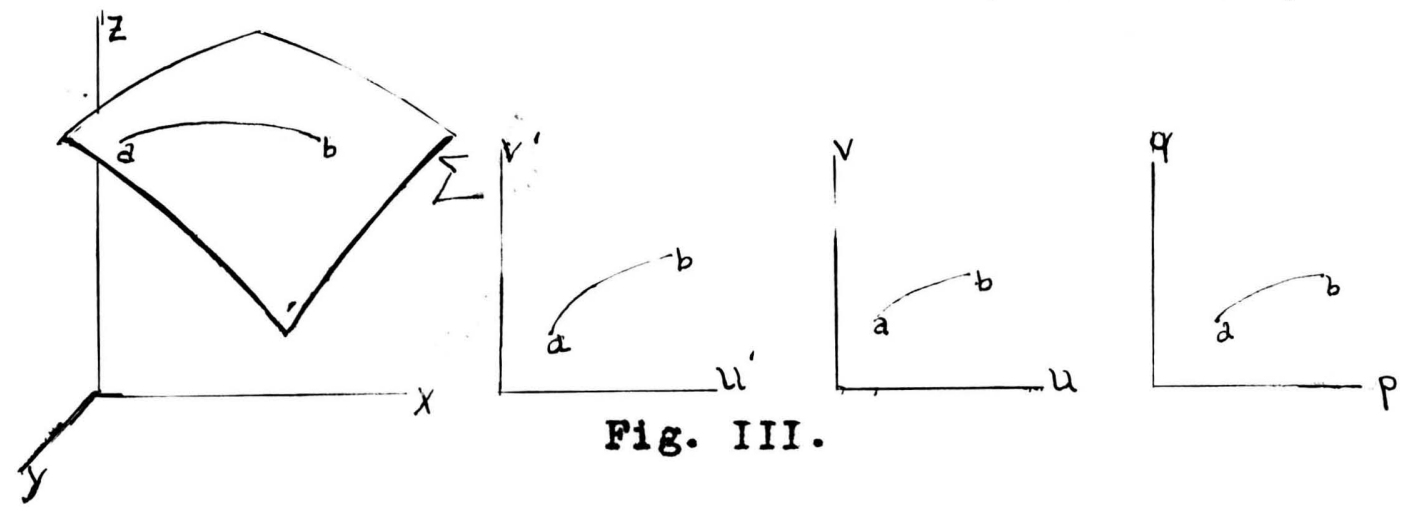

FIg. III.

11) E. Picard: op.cit., p. 8. The method by which Beltrami reaches these is entirely different from the one used here. (ii) See foot-notes, page 14. 



\section{As in the case of analytic functions, we wish to}

find the condition that

$$
\int_{d}^{b} F(w) d w
$$

be independent of the path.

NOW

$$
\begin{aligned}
& \Delta v v=\Delta u+\Delta v i \\
& d v v=d u+d v i
\end{aligned}
$$

and also

$$
\begin{aligned}
& d u=u_{p} d p+u_{q} d q \\
& d v=v_{p} d p+v_{q} d q .
\end{aligned}
$$

then

(16) $d u^{2}+d v^{2}=\left(u_{p}^{2}+v_{p}^{2}\right) d p^{2}+2\left(u p u q+v_{p} v_{q}\right) d p d q+\left(u_{q}^{2}+v_{q}^{2}\right) d q^{2}$,

but by hypothesis

$$
d u^{2}+d v^{2}=\lambda\left(E d_{p}^{2}+2 F d p d q+G d q_{q}^{2}\right)
$$

whence, factoring these two, we have

$$
(d u+d v i)(d u+d v i)=(\eta+\varphi i)\left(\eta-\varphi_{i}\right)\left(\sqrt{E} d p+\frac{F+i H}{\sqrt{E}} d q\right)\left(\sqrt{E-d}+\frac{F-i H d}{\sqrt{E}} d q\right) .
$$

therefore,

$$
d v v d u+d v i=\left(\eta+\varphi_{i}\right)\left(\sqrt{E} d p+\frac{F+i H}{\sqrt{E}} d q\right),
$$

where

$$
H=\sqrt{E G-F^{2}},
$$

and also from (16) we have

$$
\left.\left(u_{p}+i v_{p}\right) d p+\left(u_{q}+i v_{q}\right) d q=\left(\eta+\varphi_{i}\right)(\sqrt{E d})_{p}+\frac{F_{+i} H}{\sqrt{E}} d q\right) ;
$$

equating real and imaginary parts, we have

(18)

$$
u_{p} d p+u_{q} d q=\eta \sqrt{E} d p+\eta \frac{F-\varphi H A}{\sqrt{E}} d q
$$

$$
v_{p} d p+v_{q} d q=\varphi V E d p+4 \frac{F+\eta H}{\sqrt{E}} d q \text {. }
$$



20.

Equating the coefficients of $d p$ and of $d q$, we obtain

$$
u_{p}=\eta \sqrt{E} \quad, \quad v_{p}=\xi \sqrt{E}
$$

(19)

$$
u q=\frac{\eta F-\varphi H}{\sqrt{E}}, \quad v_{q}=\frac{\xi F+\eta H}{\sqrt{E}} \text {. }
$$

If we replace $d w$ under the integral sign by its value obtained in (17) and replace $F(1 / v)$ by $A(p, q)+B(p, q) i$, we have

$$
\begin{aligned}
\int_{d}^{b} F(v) d w= & \int_{d}^{b}\{A(p, q)+B(p, q) i\}(\eta+\varphi i)\left(\sqrt{E} d p+\frac{F+i H d}{\sqrt{E}} d q\right) \\
= & \int_{d}^{b}\{(\eta A(p, q) \sqrt{E}-\} B(p, q) \sqrt{E}) d p \\
& \left.\left.\left.+\left(\eta A(p, q) \frac{E}{\sqrt{E}}-\right\} A(p, q) \frac{H}{\sqrt{E}}-\right\} B(p, q) \frac{F}{\sqrt{E}}-\eta B(p, q) \frac{H}{\sqrt{E}}\right) d q\right\} \\
+ & \int_{d}^{b}\{(\} A(p, q) \sqrt{E}+\eta B(p, q) \sqrt{E}) d p \\
& +\left(\eta A(p, q) \frac{H}{\sqrt{E}}+\eta B(p, q) \frac{F}{\sqrt{E}}\right. \\
& \left.\left.\left.+\} A(p, q) \frac{E}{\sqrt{E}}-\right\} B(p, q) \frac{H}{\sqrt{E}}\right) d q\right\}
\end{aligned}
$$

In order that the integral be independent of the path it is necessary that each of the above integrals be independent of the path, hence the necessary conditions that $\int F(w) d w$ be independent of the path are that the following equations hold

$$
\begin{aligned}
& \frac{\partial}{\partial \varphi}(\eta A \sqrt{E}-\xi B \sqrt{E})=\frac{\partial}{\partial p}\left(\eta A \frac{E}{\sqrt{E}}-\varphi B \frac{E}{\sqrt{E}}-\varphi A \frac{H}{\sqrt{E}}-\eta \frac{B H}{\sqrt{E}}\right) \\
& \frac{\partial}{\partial \varphi}(\varphi A \sqrt{E}+\eta B \sqrt{E})=\frac{\partial}{\partial p}\left(\eta A \frac{H}{\sqrt{E}}+\eta B \frac{F}{\sqrt{E}}+\varphi A \frac{H}{\sqrt{E}}-\xi B \frac{H}{\sqrt{E}}\right)
\end{aligned}
$$



In partially carrying out the differentiation indicated, we obtain

$$
\begin{aligned}
& \left.\eta \sqrt{E} \frac{\partial A}{\partial q}-\xi \sqrt{E} \frac{\partial B}{\partial q}+A \frac{\partial}{\partial q} \eta \sqrt{E}-B \frac{\partial}{\partial q}\right\} \sqrt{E} \\
= & \eta \frac{F}{\sqrt{E}} \frac{\partial A}{\partial p}-\xi \frac{H}{\sqrt{E}} \frac{\partial B}{\partial p}-\varphi \frac{H}{\sqrt{E}} \frac{\partial A}{\partial p}-\eta \frac{H}{\sqrt{E}} \frac{\partial B}{\partial p} \\
& \left.+A \frac{\partial}{\partial p} \eta \frac{E}{\sqrt{E}}-B \frac{\partial}{\partial p}\right\} \frac{H}{\sqrt{E}}-A \frac{\partial}{\partial p} \frac{H}{\sqrt{E}}-B \frac{\partial}{\partial p} \eta \frac{H}{\sqrt{E}}
\end{aligned}
$$

and

$$
\begin{aligned}
& \varphi \sqrt{E} \frac{\partial A}{\partial q^{\prime}}+\eta \sqrt{E} \frac{\partial B}{\partial q}+A \frac{\partial}{\partial q} \varphi \sqrt{E}+B \frac{\partial}{\partial q} \eta \sqrt{E} \\
(23)= & \eta \frac{H}{\sqrt{E}} \frac{\partial A}{\partial p}+\eta \frac{E}{\sqrt{E}} \frac{\partial B}{\partial p}+\varphi \frac{E}{\sqrt{E}} \frac{\partial A}{\partial p}-\varphi \frac{H}{\sqrt{E}} \frac{\partial B}{\partial p} \\
& +A \frac{\partial}{\partial p} \eta \frac{H}{\sqrt{E}}+B \frac{\partial}{\partial p} \eta \frac{F}{\sqrt{E}}+A \frac{\partial}{\partial p} \varphi \frac{F}{\sqrt{E}}-\beta \frac{\partial}{\partial p} \varphi \frac{H}{\sqrt{E}} .
\end{aligned}
$$

Now since $u$ and $v$ are continuous functions of $p$ and $q$,

and

$$
u_{p q}=u_{q p}
$$

By taking this fact into account and making use of equations (19), equations (22) and (23) become (24) $\eta \sqrt{E} \frac{\partial A}{\partial \phi}-\varphi \sqrt{E} \frac{\partial B}{\partial q}=\left(\eta \frac{F}{\sqrt{E}}-\xi \frac{H}{\sqrt{E}}\right) \frac{\partial}{\partial p} A-\left(\varphi \frac{F}{\sqrt{E}}+\eta \frac{H}{\sqrt{E}}\right) \frac{\partial B}{\partial \phi}$.

and

(25) $\xi \sqrt{E} \frac{\partial A}{\partial q}+\eta \sqrt{E} \frac{\partial B}{\partial \phi}=\left(\eta \frac{H}{\sqrt{E}}+\xi \frac{E}{\sqrt{E}}\right) \frac{\partial H}{\partial p}+\left(\eta \frac{F-}{\sqrt{E}} \xi H\right) \frac{\partial B}{\partial P}$. 

Eliminating first $\frac{\partial A}{\partial \phi}$, then $\frac{\partial B}{\partial \phi}$ we obtain,

(26)

$$
E \frac{\partial B}{\partial q}=H \frac{\partial A}{\partial p}+F \frac{\partial B}{\partial p} \text {. }
$$

$$
E \frac{\partial A}{\partial q}=F \frac{\partial A}{\partial p}-H \frac{\partial B}{\partial p} \text {. }
$$

which may be put in the form

$$
\frac{\partial A}{\partial P}=\frac{E \frac{\partial B}{\partial \alpha}-F \frac{\partial B}{\partial P}}{H}
$$

$$
\frac{\partial A}{\partial \phi}=\frac{F \frac{\partial B}{\partial \phi}-C \frac{\partial B}{\partial p}}{H} .
$$

We see that the conditions that the integral

$$
\int_{d}^{b} F(v v) d v v
$$

be independent of the path are that the Beltrami equations be satisfied. Hence, in the classes of functions as in the case of the analytic functions, the conditions that the derivative be unique and definite and condition that the integral depend only on the limits of integration are the same.

$$
\begin{aligned}
& \text { If we define the function } \\
& F(v)=\Theta(r)=A(p, q)+B(p, q) i
\end{aligned}
$$

as an "analytic" function upon the surface $\sum$, whenever it satisfies the Beltrami equations for this surface (E, F, and $G$ in the Beltrami equations depend upon the particular surface in question), we may state the following theorem:

Given a function $\Theta(r)$ defined by a given surface and "analytic" on that surface, the integras $\int_{d}^{b} \Theta(r) d v /(r)$ is independent of the path. 

3. Conformal Transformation of a Surface on Itsedf.

In our study of analytic functions, we found that the demand for the existence of the derivative forced upon us both conformal and isogonat transformation. And in an examination of the proposition in which we demanded that the transformation be either conformal or isogonal we were lead to the result that the function be analytic, - that is, that the Cauchy-Riemann equations hold. (i) In these classes of functions which we are studying we should like to see if the same thing holds true, - that is, if the demand for the existence of a derivative on the surface will force upon us both conformality and isogonality. We shail attack the other problew, however, and see if the conformal or 180gonal transformation of a surface upon itself leads to the same conditions that the demand for the existence of a derivative does. Or stated in other words, if the transformation of a surfece upon itself be isogonat, do the Beltrami equations hold? In order to study this problem it is necessary to find the conditions which must be satisfied in order that the angles be conserved in the transformation. Let us first consider the general case of a transformation of one surface $\sum$ upon another $\Sigma^{\prime}$.

(1) See any work on Anaiysis. Goursat: op. cit., et al. 

Let $x, y, z$ be the rectangular coordinates of any point on the surface $\sum$. We shall suppose that the six coordinates $x, y, z ; x^{\prime}, y^{\prime}, z$ are expressed as functions of two variable parameters $p, q$ in such a way that the corresponding points of the two surfaces correspond to the same system of values of the parameters $p, q$.
(28) $\sum\left\{\begin{array}{l}x=f(p, q) \\ y=\varphi(p, q) \\ z=\psi(p, q)\end{array}\right.$
$\Sigma^{\prime}\left\{\begin{array}{l}x^{\prime}=f^{\prime}(p, q) \\ y^{\prime}=\phi^{\prime}(p, q) \\ z^{\prime}=\psi^{\prime}(p, q)\end{array}\right.$

We admit, moreover, that the functions $f, \varphi, \psi_{;} f^{\prime}, \varphi^{\prime}, \psi^{\prime}$ with their first derivatives are continuous. Let us recald the notation preifousty used.

(29)

$$
\begin{array}{ll}
E=S\left(\frac{\partial x}{\partial p}\right)^{2} & E^{\prime}=S\left(\frac{\partial x^{\prime}}{\partial p}\right)^{2} \\
F=S\left(\frac{\partial x}{\partial p} \frac{\partial x}{\partial q}\right) & F^{\prime}=S\left(\frac{\partial x^{\prime}}{\partial p} \frac{\partial x^{\prime}}{\partial q}\right) \\
G=S\left(\frac{\partial x}{\partial q}\right)^{2} & G^{\prime}=S\left(\frac{\partial x^{\prime}}{\partial q}\right)^{2} \\
& d s^{2}=E d p^{2}+2 F d p d q+G d q^{2} \\
d s^{\prime 2}=E d p^{2}+2 F^{\prime} d p d q+G^{\prime} d q^{2} .
\end{array}
$$

Let $C$ and $D$ be tro curves on the surface $\sum$, passing through a point $m$ of this surface, $C^{\prime}$ and $D^{\prime}$ the corresponding curves of the surface $\Sigma^{\prime}$, passing through the point $\mathrm{m}^{\prime}$; along the curve $C$, the parameters are functions of a single auxidiary variable $t$, and we designate the differentials by $d p$ and $d q$. In the same way along the curve the parameters are functions of another variable $t^{\prime}$, and we designate the differentials by $\delta_{p}$ and $\delta_{q}$. In the same general way, we distinguish by the letters $d$ and $\delta$ the differentials with respect to a displacement upon the curve $C$ and upon the curve $D$. 

25.
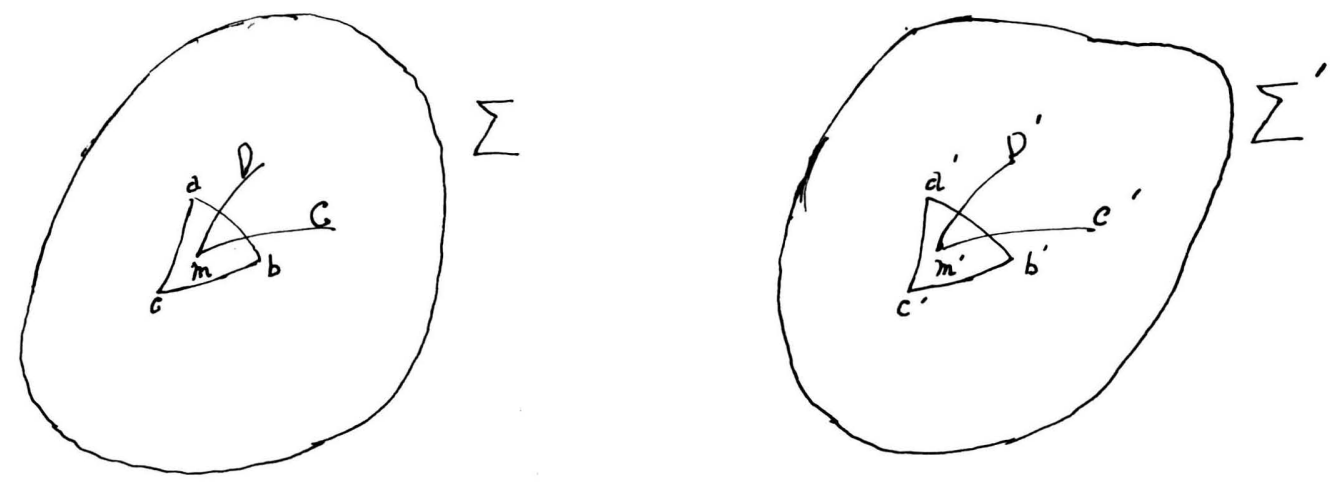

Fig. Iv.

The direction parameters of the tangent to the curve $C$ are respectively

$d x=\frac{\partial x}{\partial p} d p+\frac{\partial x}{\partial g} d q, \quad d y=\frac{\partial y}{\partial p} d p+\frac{\partial y}{\partial q} d q, \quad d z=\frac{\partial z}{\partial p} d p+\frac{\partial z}{\partial q} d q$,

likewise the directions parameters of the tangent to the

curve $D$ are respectively

$\delta_{x}=\frac{\partial x}{\partial p} \delta_{p}+\frac{\partial x}{\partial q} \delta_{q}, \quad \delta_{y}=\frac{\partial y}{\delta p} \delta_{p}+\frac{\partial y}{\partial q} \delta_{q}, \quad \delta_{z}=\frac{\partial z}{\partial p} \delta_{p}+\frac{\partial z}{\partial q} \delta_{q}$

Let $\omega$ be the angle made by the tangents to the tro curves

$C$ and $D ; \cos w$ is then given by the formula

$$
\dot{c o s} \omega=\frac{d x \delta x+d y \delta y+d z \delta z}{\sqrt{d x^{2}+d y^{2}+d z^{2}} \sqrt{\delta x+\delta y^{2}+\delta z}} .
$$

which may be written, by taking into account equations (29)

(30) $\cos \omega=\frac{E d p \delta_{p}+F\left(d p \delta_{q}+\delta p d q\right)+G d q \delta q}{\sqrt{E d_{p}^{2}+2 F d_{p} d q+G d_{q}^{2}} \sqrt{E \delta_{p}^{2}+2 F \delta_{p} \delta q+G \delta \delta^{2}}}$.

In the same way, $\omega^{\prime}$ being the angle between the tangents of the curves $C^{\prime}$ and $D^{\circ}$;

(31) $\cos \omega^{\prime}=\frac{E^{\prime} d p \delta_{p}+F^{\prime}\left(d p \delta q+\delta_{p} d q\right)+G^{\prime} d q \delta q}{\sqrt{E d_{p}^{\prime}+2 F^{\prime} \delta_{p} \delta_{q}+G^{\prime} d q^{2}} \sqrt{E^{\prime} \delta_{p}^{2}+2 F^{\prime} \delta_{p}+G^{\prime} \delta_{q}^{2}}}$. 

In order that the transformation considered may not change the value of the angle, it is necessary that $\cos \omega=\cos \omega^{\prime}$, whatever the values of $d_{p}, d_{q}, \delta_{p}, \delta_{q}$; The two members of the equation

$$
\cos ^{2} \omega=\cos ^{2} \omega^{\prime}
$$

are rational functions of the two ratios $\delta_{q}: \delta_{p}, d_{q} d_{p}$ and ought to be equal whatever the value of the two ratios. In order that this be true it is necessary that the corresponding coefficients of the two fractions be proportional, that is to say that

$$
\frac{E^{\prime}}{E}=\frac{E^{\prime}}{F}=\frac{G^{\prime}}{G}=\lambda^{2}
$$

where $\lambda$ is ar function of $p, q$. These conditions are evidently sufficient, because $\cos \omega$ is a homogeneous function of zero degree of E, F, G.

The conditions (32) may be replaced by the single relation $d s^{\prime 2}=\lambda^{2} d s^{2}$, whence

$$
d s^{\prime}=\lambda d s
$$

which expresses that the ratio of the two infinitedy small corresponding ames tends toward a limit independent of $d_{p}$ and of $d_{q}$, when these axes are diminished indefinitely. This condition makes the result almost intuitive. In proof, let us take upon the first surface an infinitely small triangle $a b c$, and let $a^{\prime} b^{\prime} c^{\prime}$ be the corresponding triangle on the second surface. Let us consider these triangles abc and $a^{\prime} b^{\prime} c^{\prime}$ as rectilinear triangles: since the ratio $a^{\prime} b^{\prime}: a b$, 

$a^{\prime} c^{\prime}: a c$, and $b^{\prime} c^{\prime}:$ bc approach the same limit $\lambda(p, q)$, in the limit the triangles abc and $a^{\circ} b^{\circ} c^{\circ}$ are similar and the corresponding angles are equal.

One sees that the two infinitedy smatl parts of the surface can be considered as similar, since the lengths of arcs are proportional and the angles are equal. It is for this reason that one often gives the name of conformal transformation to a transformation which conserves the angles!(1)

Let us now return to our problem of the transformation of a surface $\sum$ upon itself, (ii) and see if the Beltrami equations are satisfied when the transformation is isogonal.

We have given the surface $\sum$ defined by the following equations :

$$
\begin{aligned}
& x=f(p, q) \\
& y=q(p, q) \\
& z=\psi(p, q)
\end{aligned}
$$

1i) The above on the transformation of one surface upon another is a free translation from Goursat: op. cit., Vol. II., pp. 47 - 49. (A slight change in the notation has been made in order to be consistent with our other notation.) (11) This case that we are considering is not the one for which Professor Goursat finds the conditions that the transformation be isogonal, but it is evident, if the corresponding triangles abc, $a^{\prime} b^{\prime} c^{\prime}$ on the surface' $\Sigma$ are to be similar, that $d s^{\prime}$ must equal $\lambda d s$ 

where

$$
\begin{aligned}
& p=p(t) \\
& q=q(t)
\end{aligned}
$$

Let us take the function $W=u+v i$ on the surface $\sum$ where

(3ら)

$$
L=A(p \cdot q)
$$

$$
V=\beta(p, q)
$$

such that $u=e_{1}, V=c_{2}$

defines a network of isothermal lines, (ithen it follows that (36)

$$
d u^{2}+d v^{2}=\dot{\mu}\left(E d p^{2}+2 F d p d q+G d q^{2}\right)
$$

where $\mu$ is a function of $\rho$ and $q$ alone and whate $E, F$, and $G$ are defined by equations (10).

Now let us transform the surface upon itself such that this system of isothermal lines goes into another system of isothermal lines isogonally.

$$
\text { Let } u^{\prime}=r_{1}, V^{\prime}=r_{2} \quad \text { define }
$$

this second system of isothermal lines, where

$$
u^{\prime}=P(p, q)
$$

$$
V^{\prime}=Q(p, q)
$$

Since this transformation is to be isogonal,

$$
\frac{d u^{2}+d v^{2}}{d w u^{2}+d v^{2}}=\lambda^{2}
$$

(i) Any function that belongs to these ciasses must fulfil this condition, since we have always assumed that the function satisfies equations (36). 

29.

From equations (35), we have

(39)

$$
\begin{aligned}
& d_{u}=A_{p} d_{p}+A_{q} d q \\
& d v=B_{p} d_{p}+B_{q} d q,
\end{aligned}
$$

and from equations (37)

(40)

$$
\begin{aligned}
& d u u^{\prime}=P_{p} d_{p}+P_{q} d q \\
& d v^{\prime}=Q_{p} d_{p}+Q_{q} d q .
\end{aligned}
$$

If we substitute the values of $d_{2 l}$ and $d v$ obtained from (36) and the values of $d u^{\prime}$ and $d v^{\prime}$ obtained from (40) in equation $(38)$, it becomes

$$
\frac{E d d^{2}+2 F d p d q+G d_{q}^{2}}{\left(p_{p}^{2}+Q_{p}^{2}\right) d_{p}^{2}+2\left(p_{p} p_{q}+Q_{p} Q_{q}\right) \alpha p d q+\left(p_{q}^{2}+Q_{q}^{2}\right) d d^{2}}=\frac{\lambda^{2}}{\mu} .
$$

and since the transformation is isogonal, this is true whatever the values of $d p$ and $d q$, hence we have

(41) $\frac{E}{p_{p}^{2}+Q_{p}^{2}}=\frac{F}{p_{p} p_{q}+Q_{p} Q_{q}}=\frac{G}{p_{q}^{2}+Q_{q}^{2}}=\frac{\lambda^{2}}{\mu}$.

From which we obtain,

$$
\frac{E G-F^{2}}{F^{2}}=\frac{\left(p_{p} Q_{q}-Q_{p} P_{q}\right)^{2}}{\left(P_{p} P_{q}+Q_{p} Q_{q}\right)^{2}}
$$

Taking the square root of both sides of the equation, we have

$$
\frac{H}{F}=\frac{P_{p} Q_{q}-Q_{p} P_{q}}{P_{p} P_{q}+Q_{p} Q_{q}}
$$



30.

where $H=\sqrt{E G-F^{2}}$

or

(42) $H\left(P_{p} P_{q}+Q_{p} Q_{q}\right)=F\left(P_{p} Q_{q}-Q_{p} P_{q}\right)$

If we take

$$
\frac{E}{p_{p}^{2}+Q_{p}^{2}}=\frac{F}{p_{p} P_{q}+Q_{p} Q_{q}}
$$

and solve first for $P_{q}$, and then for $Q_{q}$, we have

(43) $\quad E P_{q}=\frac{F\left(p_{p}^{2}+Q_{p}^{2}\right)-E Q_{p} Q_{q}}{p_{p}}$,

and

1441 $E Q_{q}=\frac{F\left(p_{p}^{2}+Q_{p}^{2}\right)-E p_{p} P_{q}}{Q_{p}}$

From (42) we have

and

$$
Q_{q}=\frac{P_{q}\left(F Q_{p}+H P_{p}\right)}{F P_{p}-H Q_{p}}
$$

$$
P_{q}=\frac{Q_{q}\left(F P_{p}-H Q_{p}\right)}{F Q_{p}+H P_{p}}
$$

substituting the value of $P_{q}$ in (43) and the value of $P_{2}$, 

in (44), and simplifying, equations (43) and (44) become respectively,

and

$$
E Q_{q}=F Q_{p}+H P_{p}
$$

$$
E P_{q}=F P_{p}-H Q_{p} \text {. }
$$

which are the Beltrami equations.

Hence we see that the demand that the transfornation

of a surface upon itself such that isothermal lines go into sisothermal lines be isogonal forces upon us the Beltrami equations.

This demand, namely, the demand that the transformation which carries isothermal lines into isothermat lines be isogonal, is exactly the same demand that we made in the isogonal transformation of the plane upon itself; there, too, we demanded that isothermal lines go into isothermal lines by means of the isogonat transformation.

$$
\text { In order to make the above work of the transform- }
$$
ation of a surface upon itself less format, tet us state just what transformations we have considered, and what has been the nature of each. We have given the surface $\sum$ defined by equations (34), and the parameter plane - the $p, q$ - plane.
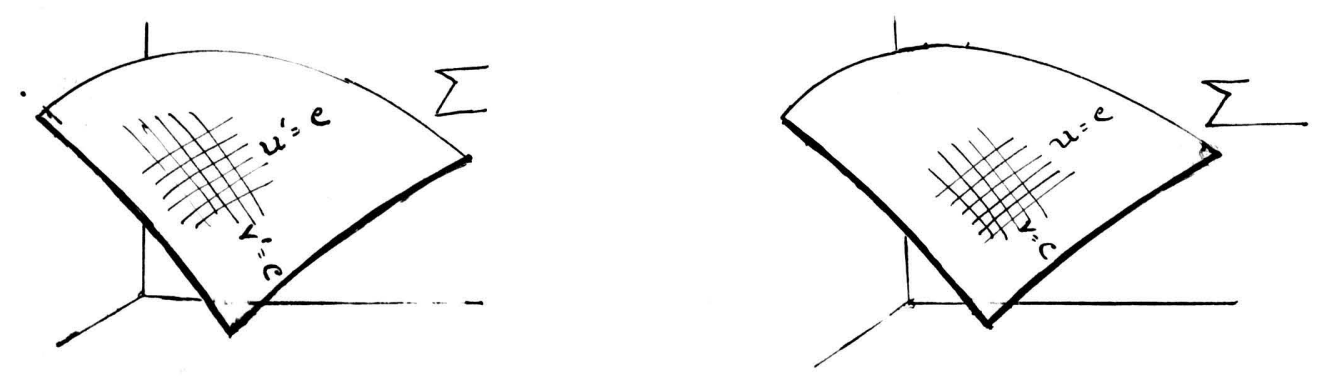


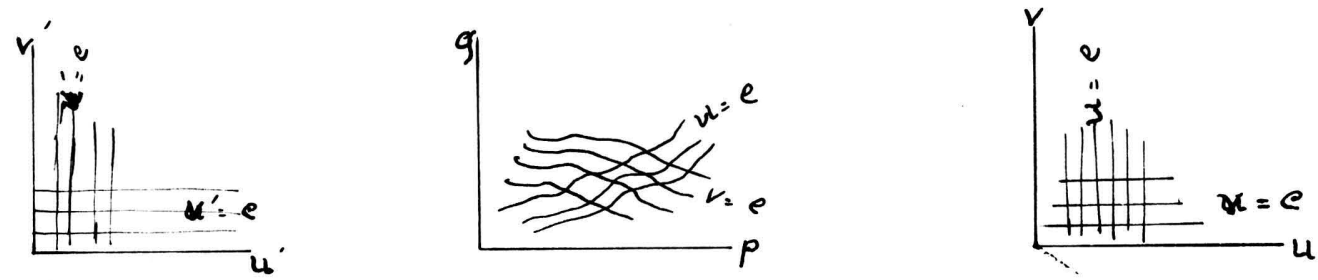

Fig. V.

and we had defined some transformation

$$
\begin{aligned}
& u=A(p, q) \\
& v=B(p, q)
\end{aligned}
$$

(which may be regarded as a non-conformat transformation of the $p, q$-plane upon the $u, v-p$ lane) such that $u=C$, and $V=C_{2}$ form a network of 180 thermal lines; and we demand that the isogonal transformation of the surface upon itself be such that

$$
\begin{aligned}
& u^{\prime}=P(p, g)=r_{1} \\
& v=Q(p, g)=r_{2}
\end{aligned}
$$

give rise to another system of isothermal. Lines on the surface $\sum$. (The equations $u^{\prime}=P_{(p, q), V^{\prime}=Q(p . q)}$ may also be regarded as defining a non-conformal transformation of the plane upon itself.)

This transformation, considered as a transformation of the $u, v$-plane upon the $u^{0}, v^{0}$-plane, is the ordinary conformal transformation of a plane upon itself. Hence $W^{\prime}=2 l^{\prime}+v_{i}^{\prime} \quad$ is an ordinary analytic function of $v V=22+v i^{\prime}$, however, we must bear in mind that the $u, v-p$ tane and the $u^{\bullet}, v^{0}$-plane are not parameter planes, but planes which are 

33.

stretched and distorted until they fit upon the surface $\sum$. It is for this reason that the transformation given above defines a conformal transformation of the surface upon itself. 

Section III.

Cauchy's Theorems, Taylor's and Laurent's Series, and other Theorems.

1. Continuous Functions.

Let us assume, as in the preceding work, that we have a surface $\sum$ defined by the equations,

$$
\begin{aligned}
& x=f(p, q) \\
& y=\varphi(p, q) \\
& z=\psi(p, q),
\end{aligned}
$$

and that we have a function $w(r)$ of the class( $i)$ that we are considering, where

$$
w=u+v i=u(p, q)+v(p, q) i
$$

and such that

$$
d u^{2}+d v^{2}=\lambda\left(E d d^{2}+2 F d p d q+G d q^{2}\right),
$$

then if

$$
r=p+q i \quad \text {, and } v v=v v(r) \text {, }
$$

$W(r)$ will be said to be a continuous function of $r$ if the moduius of the difference $w(r+h)-w(r)$ approaches zero as the modulus of $h$ approaches zero, that is, if

$$
|v v(r+h)-v v(r)|<\epsilon
$$

whenever

$$
|h|<\eta
$$

where $t$ is chosen first.

(i) Professor Picard calls these classes of functions "functions complexes de seconde espece". Cf. Picard: op. cit., vol. II., p. 541 . 



\section{Regular Functions.}

A function $V(r)$ will be said to be regular in a region $A$ on the surface $\sum$, if it satisfies the following conditions:

1) For every point $r$ of the region A, there corresponds a definite vatue $W(r)$.

2) $W(r)$ is a continuous function of $r$.

3) $W(r)$ admits of a uniquely determined derivative ${ }^{(i)}$ at every point of the region $A$.

3. The Integral taken along a Contour - Cauchy's Fundamentad Formula.

$$
\text { Let } \Theta(r) \text { and } W(r) \text { be functions of the classes }
$$

that we are studying and regular within the region $A$, where

$(45)$

$$
\Theta(r)=A(p, q)+B(p, q) i
$$

and

$$
W(r)=u(p, q)+V(p, q) i
$$

Let us now consider the integral

$$
\int_{a b c b a} \Theta(r) d v(r)=\int_{a b c} \Theta(r) d v v(r)+\int_{c b^{\prime} d} \Theta(r) d w(r)
$$

(i) By derivative here we mean derivative in the sense in which we have been using it in regard to these classes of functions, i.e., $\quad d v^{\prime} / d w$ function of the same class.

(ii) $\int \Theta(r) d w(r)$ is really $\left.\int \Theta(r) d r\right)$, if we mean by $d r$ in these integra1s, not $d_{p}+d q_{i}$, but $\left(\eta+\varphi_{i}\right)\left(\sqrt{E d_{p}+} \frac{F_{+i}}{\sqrt{E}} d t d q\right)$. 



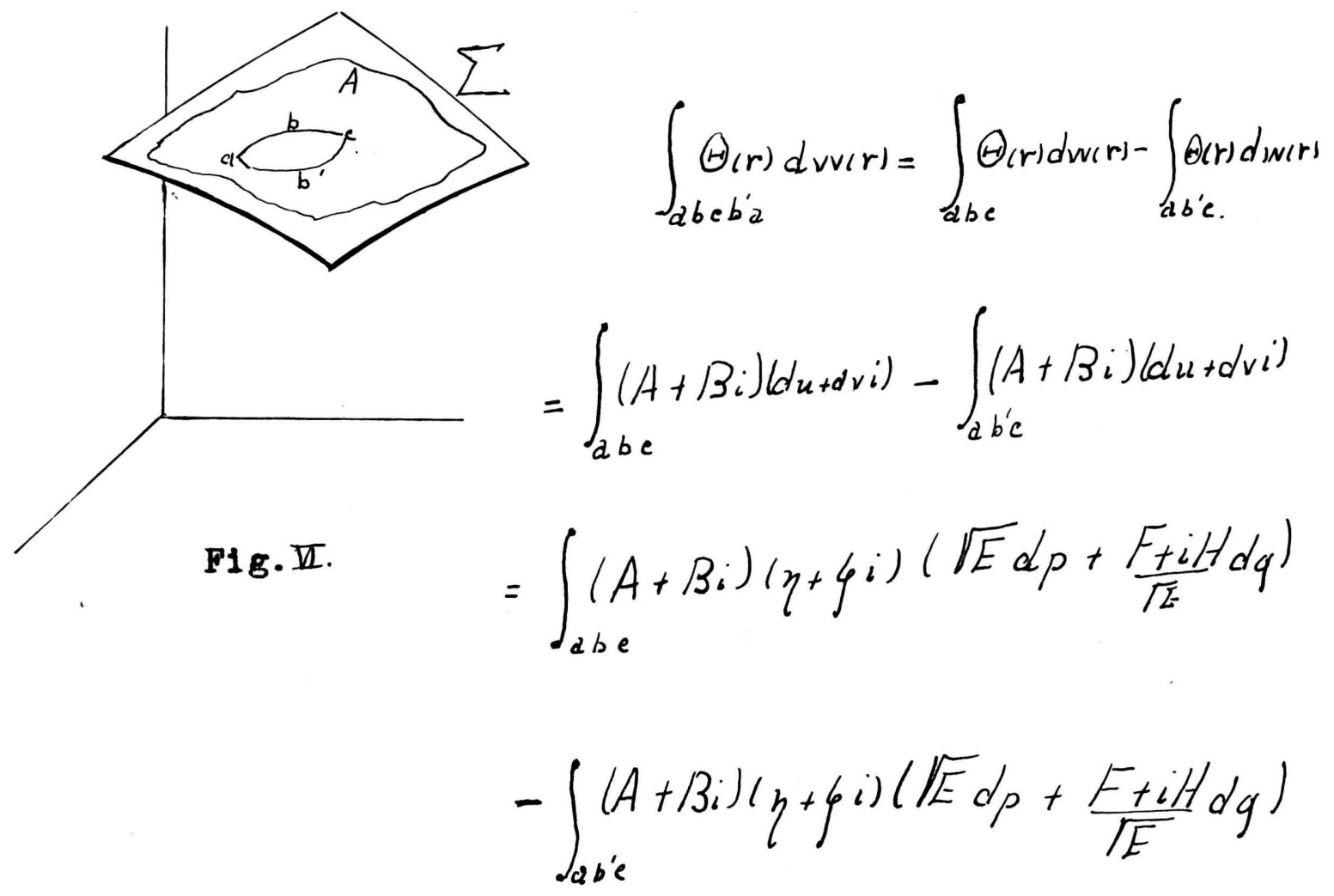

But we found ${ }^{(i)}$ that the condition that the integral be independent of the path is that the Beltrami equations be satisfied, therefore, since $\Theta(r)=A(p, q)+\beta(p, q) i^{\prime}$ is a function of the class that we have defined, the following equations are satisfied

$$
\begin{aligned}
& \frac{\partial B}{\partial p}=\frac{E \frac{\partial A}{\partial g}-F \frac{\partial A}{\partial \rho}}{H} . \\
& \frac{\partial B}{\partial q}=\frac{F \frac{\partial A}{\partial q}-G \frac{\partial A}{\partial p}}{H},
\end{aligned}
$$

(i) CP. Sec. II., 3 

and hence

$$
\begin{aligned}
& \int_{d b c}\left(A+B_{i}\right)\left(\eta+y_{i}\right)\left(\sqrt{E} d p+\frac{F+i H}{\sqrt{E}} d q\right) \\
= & \int_{d b^{\prime} c}\left(A+B_{i}\right)(\eta+\varphi i)\left(\sqrt{E} d p+\frac{F+i H}{\sqrt{E}} d \phi\right),
\end{aligned}
$$

therefore

(46) $\int_{d b c b^{\prime} d} \Theta(r) d w(r)=\int_{d b e} \Theta(r) d w\left(r-\int_{d b^{\prime} e} \Theta(r) d w(r)=0\right.$.

Hence in these classes of functions, as in the analytic functions, the integrat of a regular function taken around a closed path equal zero, and therefore for these classes of functions we have the following

Theorem: If the functions $\Theta(r)$ and $w(r)$ are "ana1yt1c" on the surface $\sum$ and are regular on the contour $C$, and within the region bounded by the contour, the integral $\int \Theta(r) d v v(r)$ taken along this contour is equat to zero.

4. Cauchy's Integral Theorem.

Again take $\Theta(r)$ and $w(r)$ regular functions withIn the region $A$ on the surface $\sum$, then the function $\frac{\Theta(r)}{w(r)-w(\mu)}$ is regular within the region $A$ except at that point on the surface $\sum$ which corresponds to the point $r=\mu$ in the $p, q$-plane. 

38.

Let us now consider the integral $\int_{c} \frac{\theta(r) d w(r)}{w(r)-w / \mu)}$

where the contour C lies entirely in the region $A$. Since $\Theta(r)$ is continuous in the region $A$,

$$
\Theta(r)=\Theta(\mu)+\epsilon
$$

where $\epsilon$ depends on $\rho$, the radius of the circle $C^{\prime}$ corresponding in the $u, v$-plane to the contour $\mathrm{C}^{\prime}$ on the surface $\sum$,

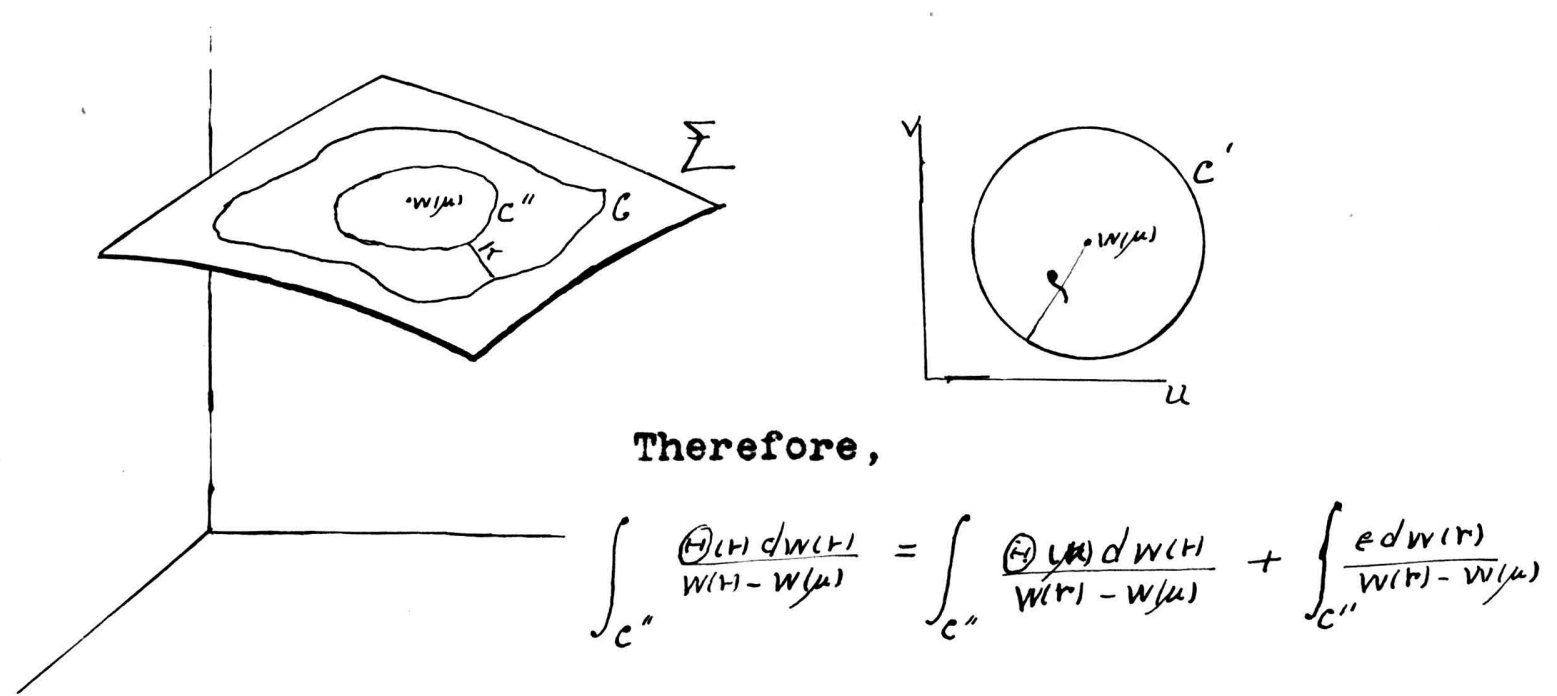

The integral $\int_{c^{*}} \frac{\theta(r) d w(r)}{w(r)-w(\mu)} \quad$ can be easily calculated.

Let

$$
\begin{aligned}
w(r)-v v(\mu) & =\int(\cos \theta+i \sin \theta) \\
d w(r) & =\rho(-\sin \theta+i \cos \theta) d \theta
\end{aligned}
$$

Substituting these values in the integral above, we have

$$
\int_{c^{\prime \prime}} \frac{\Theta(\nu) d v(r)-v(r)}{v v(\mu)}=\Theta(\mu) \int_{0}^{1 \pi} \frac{i}{f(\cos \theta+i \sin \theta)} d \theta=2 \pi i \theta(\mu)
$$

and $\int_{c^{\prime}} \frac{\epsilon d w(r)}{w(r)-w(\mu)} \quad$ is independent of the radius $\rho$, 

hence, since $\theta_{(r)}$ is continuous, we can by choosing $\rho$ as small as we please, make $\epsilon$ as small as we please, therefore

$$
\int_{c^{\prime \prime}} \frac{\epsilon d w(r)}{w(r)-w / \mu)}
$$

must equal zero.

Hence, we have

(47) $\Theta(\mu)=\frac{1}{2 \pi i} \int_{c} \frac{\theta(r) d w(r)}{w(r)-2 v(\mu)}$.

If we connect $C$ and $C^{-}$by the path $K$ and consider the integral taken along $C, K$, and $C^{\prime} '$, since the function is regular within the region bounded by this curve, the integral by Cauchy's fundamentat formula equals zero. Hence, since the two integrations across $K$ are in the opposite direction and annul each other, the integral along C." equals the integral along $C$, therefore we have

$$
\Theta(\mu)=\frac{1}{2 \pi i} \int_{e} \frac{\Theta(r) d w(r)}{w(r)-w / \mu)}=\frac{1}{2 \pi i} \cdot \int_{C} \frac{\theta(r) d w(r)-v(\mu)}{w(r)} .
$$

This is the analogon of the Cauchy integral fornula for the analytic functions, and it expresses the value of the function $\Theta(r)$ at any interior point $\mu$, in terms of the values of the function along the contour $C$. The above result may be stated as follows:

Theorem: If the functions $\Theta(r)$ and $v v(r)$ be "anaiytic" on the surface $\sum$, and if they be reguiar within the 

region $A$ on the surface $\sum$, then

$$
\Theta(\mu)=\frac{1}{2 \pi i} \cdot \int_{e} \frac{\Theta(r) d v(r)}{w(r)-N(\mu)}
$$

where C is 8 contour which lies whotly within the region A and which encloses the point $v(u)$.

\section{Expressions for the Derivatives.}

Let $\mu+\Delta \mu$ be a point very near to $\mu$, such that the point on the surface corresponding to $\mu+\Delta \mu$ will be within the "contour"c' (Fig. $\mathrm{ZII}$ ), we have then by the preceding equation (47)

$$
\Theta(\mu+\Delta \mu)=\frac{1}{2 \pi i} \int_{C} \frac{\Theta(r) d w(r)}{w(r)-v v(\mu+\Delta \mu)}
$$

and

$$
\Theta(\mu+\Delta \mu)-\Theta(\mu)=\frac{1}{2 \pi i} \cdot \int_{e}^{\Theta(r) d w(r)} \frac{1}{w(r)-v(\mu+\Delta \mu)}-\frac{1}{2 \pi i} \cdot \int_{e} \frac{\theta_{(r) d w(r)}(r)-w(\mu)}{w(}
$$

dividing through by $w(\mu+\Delta \mu)-w(\mu)$ (i) we have

$$
\begin{aligned}
& \frac{\Theta(\mu+\Delta \mu)-\Theta(\mu)}{w(\mu+\Delta \mu)-w(\mu)}=\frac{1}{2 \pi i} \cdot \int_{C} \frac{\Theta(r) d w(r)}{[v(r)-w(\mu)][w(r)-w(\mu+\Delta \mu)]} \\
& =\frac{1}{2 \pi i} \int_{C} \frac{\Theta(r) d w(r)}{[W(r)-v v(\mu)]^{2}}+\frac{1}{2 \pi i} \cdot \int_{C} \frac{\theta(r)[w(2 x+\Delta \mu)-w(\mu)] d w(r)}{[W(r)-v(\mu)]^{2}[w(r)-v v(\mu+\Delta \mu)} .
\end{aligned}
$$

(i) $\frac{d \Theta}{d w}$ is the only kind of derivative that we have assumed to exist for these functions. 

41.

Taking the limit, we have

$$
\begin{aligned}
& \left.\frac{d !}{d w}\right]_{\mu}=\lim _{\Delta \mu=0} \frac{\Theta(\mu+\Delta \mu)-\Theta(\mu)}{w(\mu+s \mu)-v(\mu)}
\end{aligned}
$$

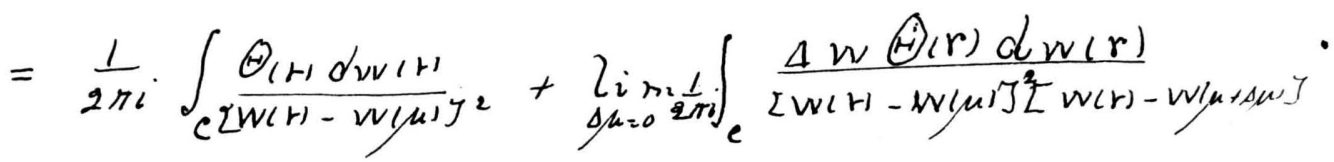

We wish to find

$$
\lim _{\Delta \mu=0} \int_{c} \frac{[v v(\mu+\Delta \mu)-v v(\mu)] \Theta(r) d w(r)}{\left[v(r)-v v(\mu) y^{2}[w(r)-v v(\mu+\Delta \mu)]\right.} .
$$

Let $M$ be the upper limit of $\Theta(r)$ along the path $C$, and $\delta$ the shortest distance of the point $v v / \mu$ ) from the contour C, then

$$
\begin{aligned}
& \lim _{4 \mu=0}\left|\int_{e} \frac{L w(\mu+\Delta \mu)-v v(\mu)] \Theta(r) d v v(r)}{[v v(r)-v v(\mu)]^{2}[v v(r)-v v(\mu+\Delta \mu)}\right| \\
& \leq \lim _{\Delta \mu=0} \frac{M L|\Delta W|}{\delta^{3}}=0
\end{aligned}
$$

hence

$$
\left.\frac{d \Theta}{d w}\right]_{\mu}=\frac{1}{2 \pi i} \cdot \int_{c} \frac{\Theta(H) d w(r)}{[W(t)-w / \mu)]^{2}} .
$$

We can in the same way show that

(48) $\frac{d^{2} \Theta}{\left.d^{2} v\right]_{\mu}}=\Theta^{\prime \prime}=\frac{1.2}{2 \pi i} \int_{e} \frac{\Theta(r) d w(r)}{\left\langle w(r)-v(\mu) J^{3}\right.}$,

and in general that

$$
\left.\frac{d^{N} \Theta}{d w^{n}}\right]_{\mu}=\Theta^{N}(\mu)=\frac{N^{\prime}}{2 \pi i} \int_{c} \frac{\Theta(r) \alpha v v(r)}{[v(r)-v v \mid \mu) T} N+1 .
$$



We see then that if $\Theta(r)$ and $v v(r)$ are "analytic functions" of the surface $\sum$, and regular within the region $A$, that the number of successive derivatives is unlimited. Hence, we see that for these classes of functions, as welt as for the ordinary analytic functions, the existence and continuity of the first derivative forces the existence and continuity of a+l the other derivative.

6. Taylor's Series.

Theorem: Let $\Theta(r)$ and $w(r)$ be analytic functions of the surface $\sum$, and reguiar on the contour" $C$ and in the region bounded by this" contour" with center at $v(\alpha)^{(1)}$; then the vaiue of this function at any point $W(\mu)$ taken within the "contour" C is equa 1 to sur of the convergent series;

$$
\begin{aligned}
& \Theta(\mu)=\Theta(\alpha)+\Theta_{w}^{\prime}(\alpha)[w(\alpha)-w(\alpha)]+\Theta^{\prime \prime}(\alpha) \frac{[w(\mu)-w(\alpha)]^{2}}{1.2} \\
& +\ldots+\ldots+\frac{\Theta_{w}^{N}(\alpha)[w(\mu)-w(\alpha)]^{N}}{1.2 .}+\cdots
\end{aligned}
$$

In order to prove this let us suppose that $\Theta(r)$ and $w(r)$ are reguiar on the "contour" and in the region bounded by it. From Cauchy's integral theorem we have:

(i) The expression 'region bounded by a "contour " $c$ with center at $v(\alpha)$ 'means a region bounded by a "contour $c$ on the surface $\sum$ which corresponds to a region in the $u, v$ plane bounded by a circle $C^{\prime}$ with center at $v v(\alpha)$. In the following theorems we shall use this same expression with the same meaning. 

43.

$\Theta(\mu)=\frac{1}{2 \pi i} \int_{c} \frac{\Theta(r) d w(r)}{w(r)-v v(\mu)}$.
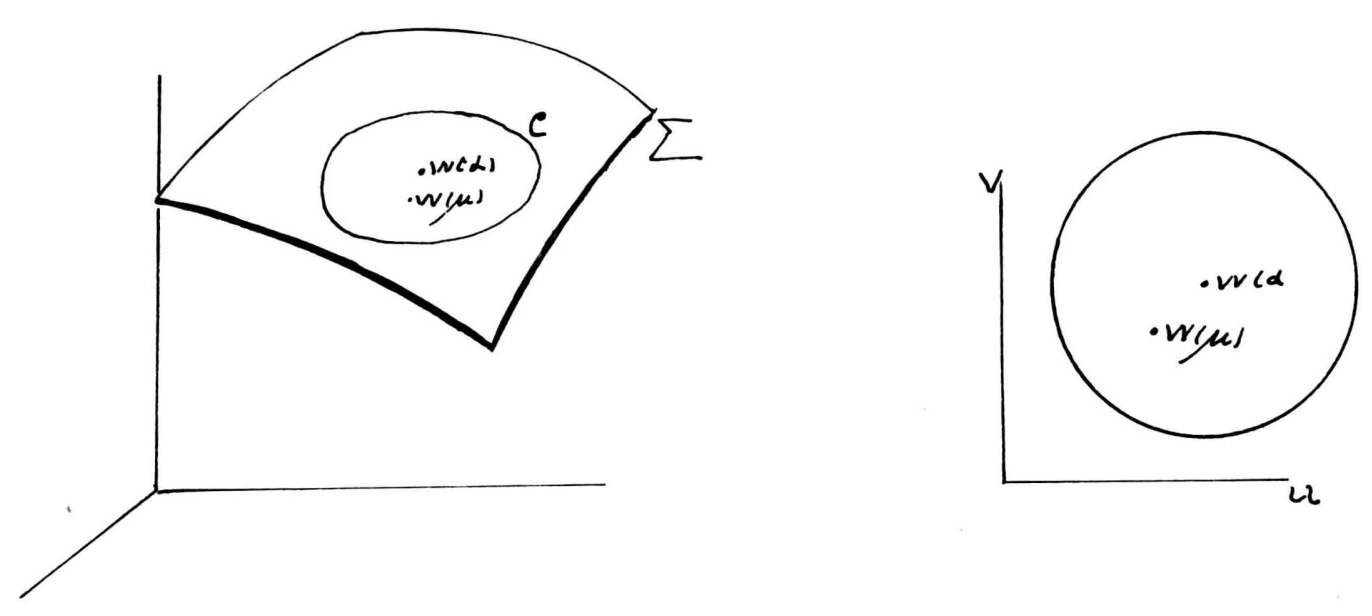

Fig. IIII.

Now let us write $\frac{1}{w(r)-v v(\mu)}$ in the following form:

$\frac{1}{w(r)-v v(\mu)}=\frac{1}{w(r)-v v(\alpha)-2 v v(\mu)-w(\alpha)]}=\frac{1}{w(1)-v v(\alpha)}\left(\frac{1}{1-\frac{w(\mu)-v v(\alpha)}{w(1)-v v(\alpha)}}\right)$.

Expanding the above expression for $\frac{1}{w(t)-w(\mu)}$, we have

$$
\begin{aligned}
& \frac{1}{w(r)-v N(\mu)}=\frac{1}{w(r)-v v(\alpha)}+\frac{w(\mu)-w(\alpha)}{[w(1)-v v(\alpha)]^{2}}+\cdots \cdots \\
& -\cdots+\frac{[w(\mu)-w(\alpha)]^{N}}{\left[v v(r)-v v(\alpha) \zeta^{N+1}\right.}+\frac{[v v(\mu)-w(\alpha)]^{N+1}}{[v(r)-w)(\mu)[v v(r)-w(\alpha)]^{N+1}}
\end{aligned}
$$

In the above expression for $\Theta(\mu)$, replacing $\frac{1}{w(r)-w(\mu)}$

by this value, we have 

$(4 q) \Theta(\mu)=J_{0}+J_{1}[v v(\mu)-v v(\alpha)]+J_{2}[v v(\mu)-v v(\alpha)]_{+}^{2}-$. $\ldots+\ldots+J_{N}[w(\mu)-w(\alpha)]^{N}+P_{N}$,

where the coefflcients $J_{0}, J_{1}, \ldots . J_{i}$ and $R_{N}$ have the following values:

$$
J_{0}=\frac{1}{2 \pi i} \cdot \int_{e} \frac{\Theta(r) d v v(r)}{W(r)-v v(\alpha)}, \quad J_{1}=\frac{1}{2 \pi i} \int_{e} \frac{\Theta(r) d n(r)}{[W(r)-v v(\alpha)]^{2}}
$$

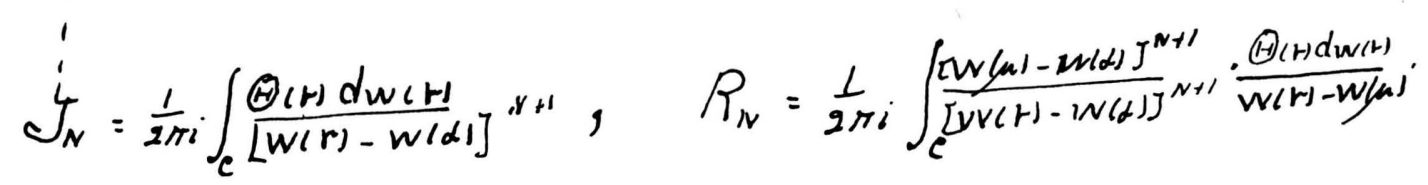

We should now like to show that when $\underline{n}$ increases inderinitely, $t_{N}$ approaches zero, that is, that the above series is convergent. Let $M$ be the maximun value of $/ \Theta(r) /$ along the path $C, P$ the maximum distance of $v(N)$ from the contour, or the radius of the oorresponding circle in the $u, v-$ plane, and $\rho=|w(\mu)-w(\alpha)|$, then $|w(r)-w| \mu|| \leq P_{-} \rho$ and $L$ the integral $\int_{e} d w(r)$, therefore

$$
\left|P_{N}\right|<\frac{1}{2 \pi}\left(\frac{\rho}{p}\right)^{N+1} \cdot \frac{M L}{P-\rho} \leq \frac{1 \xi}{P-\rho}\left(\frac{\rho}{p}\right)^{N+1}
$$

where $K$ is some constant. Since the factor $P / p$ tends toward zero, when $\underline{n}$ is increased indefinttely, $R_{N}$ approaches zero, therefore

$$
\Theta(\mu)=J_{0}+I_{1}\left(v v(\mu)-v(\alpha) \ldots \ldots+J_{N}(w / \mu)-w(\alpha)\right]^{N}+\ldots
$$



Comparing the coefficients $J_{0}, J_{1} \ldots \ldots . J_{N} \ldots$ with the expressions in equations (47) and (48) we see that

$$
\begin{aligned}
& \Theta(u)=\Theta(\alpha)+\Theta^{\prime}(\alpha)[w(\mu)-v(\alpha)]+\frac{\Theta(\alpha)}{1,2}[v / v(\mu)-v V(\alpha)]^{2} \\
& +\ldots \ldots \ldots+\ldots,(\alpha) \frac{[w(\mu)-w(\alpha)]^{N}}{1, \ldots \ldots N}+\ldots
\end{aligned}
$$

Hence our theorem is proved.

The analogon of this in the analytic functions has been called the Cauchy-Taylor development of a function of a complex variable. The circle of convergence in the case of analytic functions extended until it touched the singular point of the function. We shail discuss later, under the topic - Region of Convergence, the region within which the series just discussed converges.

\section{Laurent's Series:}

Laurent made a very important extension of the Cauchy-Taytor development of anaiytic functions within regions for which the Cauchy-Taylor development does not hold. Let us see if we can make an analogous extension of the Cauchy-Taytor development for these classes of functions with which we are interested.

Let $C$ and $C^{\prime}$ be two"concentric contours"(i)

(1) These "concentric contours" on the surface $\sum$ correspond to two concentric circles in the $u+v$-plane with their center at $N(\alpha)$. 

46.

on the surface $\sum$ with center at $v v(\alpha)$ and let $C^{\prime}$ be the inner of these "contours". Take the functions $\Theta(r)$ and $\mathrm{W}(r)$ which are regular throughout the region included between these "contours and also on the "contours" $C$ and $C$ '. The function

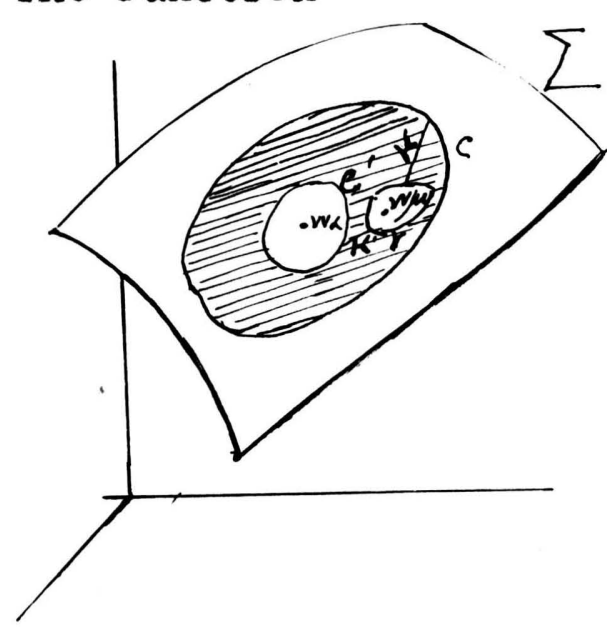

Fig. $\not X$.
$\frac{\Theta(r)}{W(r)-v v(\mu)}$

is regular within the included region, except at the point $w(\mu)$ (Choose vvlu) so that it lies withIn the region bounded by the "contours " $C$ and $C^{\prime}$ ). If we surround by a small "contour" $r$ (which corresponds to a circle in the

$U L, V$-plane with center at $w(\mu)$ ) and draw lines $K$ and $K^{\prime}$ connecting $\gamma$ to $C$ and $C^{\prime}$ respectively, the function

$$
\frac{\theta(r)}{w(r)-w(\mu)} \text { within the shaded part of the figure is }
$$

everywhere regular and the Cauchy fundamental formula holds, hence

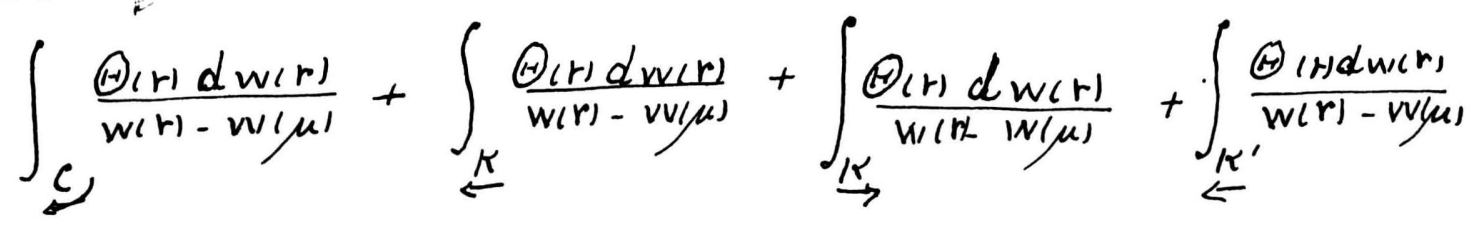

$$
\begin{aligned}
& +\int_{r^{\prime}} \frac{\Theta(r) d w(r)}{w(r)-r v(\mu)}+\int_{e^{\prime}} \frac{\Theta(r) d w(r)}{w(r)-W(\mu)}+\int_{Y_{G}} \frac{\Theta(r) d w(r)-\mid w / \mu)}{W(r)}=0 .
\end{aligned}
$$



The two integrals across $K$, since they are in the opposite direction, annul each other, and also, for the same reason, the integrals across $K^{\prime}$ annul each other, and the Cauchy integrel formula applies to the integral around $r$, hence we have

$$
\Theta(\mu)=\frac{1}{2 \pi i} \int_{\underline{e}_{1}} \frac{\Theta(r) d w(r)}{w(r)-w(\mu)}+\frac{1}{2 \pi i} \int_{e^{\prime}} \frac{\Theta(r) d w(r)}{w(\mu)-w(r)}
$$

the integrats $C$ and $C^{\prime}$ now being taken in the same sense. We see that the Cauchy Taylor development applies to the first integrat, and we have

$$
\frac{1}{2 \pi i} \cdot \int \frac{\Theta(r) d w(r)}{w(r)-w(\mu)}=J_{0}+J_{1}[w(\mu)-w(\alpha)]+J_{2}[w(\mu)-w(\alpha)]^{2}+\ldots
$$

where $J_{0}, J_{1} J_{2} \ldots \ldots J_{N}$ have the same vatues which they had in the preceding paragraph.

Now we wish to find the value of the integral

$$
\frac{1}{2 \pi i} \int_{e^{\prime}} \frac{\Theta(r) d w(r)}{v V(u)-v v(r)}
$$

In order to do this let us replace $\frac{1}{w / u)-w(r)}$ in the above integral by the following expression:

$$
\begin{aligned}
& \frac{1}{w(\mu)-v v(r)}=\frac{1}{w(\mu)-w(\alpha)}\left(\frac{1}{\left.1-\frac{w(r)-v v(\alpha)}{w(\mu)-w(\alpha)}\right)}=\frac{1}{w(\mu)-w(\alpha)}+\frac{w(r)-w(\alpha)}{[w / \mu 1-w(\alpha)]^{2}}\right. \\
& +\ldots+\frac{[w(r)-w(\alpha)]^{N-1}}{[v(v)-w(\alpha)]^{N}}+\frac{[w(r)-w(\alpha)]^{N}}{[w(\mu)-w(r)][v w(\mu)-w(\alpha)]^{N}}
\end{aligned}
$$



We then have

$151)$

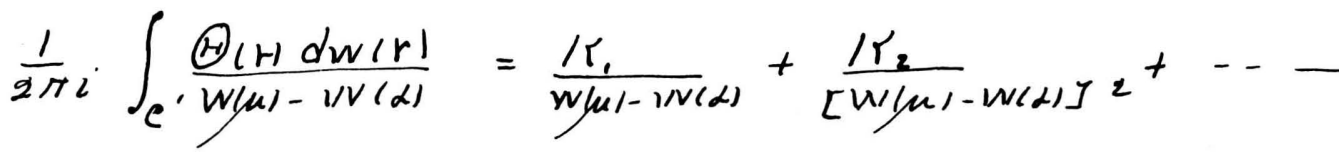

$$
\begin{aligned}
& \cdots+\frac{K_{N}}{2 w(u)-w(\alpha)]^{N}}+\frac{1}{2 \pi i} \int_{\left.\left.c^{\prime}[w(r)-w(\alpha)]\right]^{N} \theta_{(r)} d v v(r)\right][w / \mu)-1 N(\alpha)^{N}}^{[w(r)}
\end{aligned}
$$

where

$$
K_{j}=\frac{1}{2 \pi i} \int_{c^{\prime}}\left[w(r)-w(\alpha]^{j} \Theta(r) d w(r) .\right.
$$

We wish to show that the integral of the remainder term approaches zero as $\underline{\mathrm{n}}$ increases indefinitely.

Let $M^{\prime}$ be the maximun absolute value of $\Theta(r)$ along the path $C^{\cdot}, R^{\prime}$ the radius of the circle in the $l, V$-plane corresponding to the "contour" $C^{\prime}$, and $\rho=|v(\mu)-v v(\alpha)|$, then $R^{\prime}<\rho$. The absolute value of the integral is less than

$$
\frac{1}{2 \pi}\left(\frac{R^{\prime}}{\rho}\right)^{N} \frac{N^{\prime}}{\rho-R^{\prime}} 2 \pi R^{\prime}=\frac{N I^{\prime} R^{\prime}}{r-R^{\prime}}\left(\frac{R^{\prime}}{\rho}\right)^{N}
$$

but since $R^{\prime}<\rho$, the factor $\left(\frac{R^{\prime}}{\rho}\right)^{N}$ approaches zero as n increases indefinitely, therefore the integral of the remainder term approacbes zero as $\underline{n}$ becomes indefinite $\perp y$ large, and hence the series

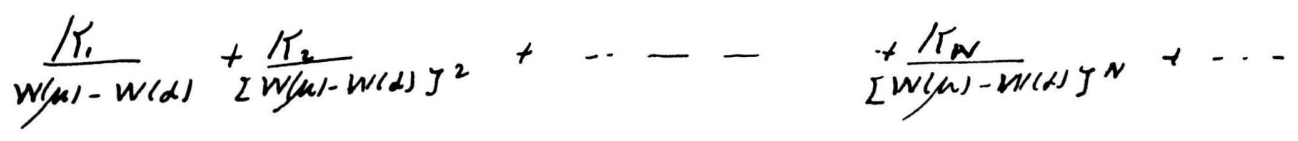

converges, and 

49 .

Or, changing our notation, we have

$(s-2) \Theta(r)=\sum^{0 \leqq j \leqq+\infty} J_{j}[w(r)-v v(\alpha)]^{j}+\sum^{1 \leqq j \leqq \infty} \frac{1}{h_{j}} \frac{1}{[v(r)-w(\alpha)]^{j}}$.

Therefore we can state the analogon of Laurent's theorem for this class of functions as follows:

Theorem: If war) be any point in the region bounded by the "contours" c and c' with centre at $k x(*)$ within which $\Theta(r)$ and weer) are regular, then $\Theta(r)$ can be expanded in the form:

$$
\Theta(r)=\sum^{0 \leqq j \leqslant+\infty} J_{j}[w(r)-w(\alpha)] j+\sum^{1 \xi j \leq \infty} / r_{j} \frac{1}{[w(r)-w(\alpha) j},
$$

Where

$$
\begin{aligned}
& J_{j}=\frac{1}{2 \pi i} \int_{c} \frac{\Theta(r) d w(r)}{[v(r)-1 / r(\alpha)] j+1} \\
& Y_{j}=\frac{1}{2 / T} \cdot \int_{c}[v v(r)-1 v(\alpha)] j^{-1} \Theta(r) d v(r) .
\end{aligned}
$$

8. Liouvilie's Theorem.

Theorem: A function $\Theta(r)$ which is regular for all finite values of $r$ and which is everywhere limited is a constant.

If $\Theta(r)$ is regular for ali finite values of $r$, it is developable in the Cauchy-Taylor expansion. Any coefficient $a_{N}$ of the term $[v r(r)-v v(\alpha)]^{N}$ is given by the following expression:

$$
a_{N}=\frac{1}{2 \pi i} \int \frac{\theta(\xi) d v(r)}{2 v(\zeta)-v v(\alpha))^{v+1}}
$$



50.

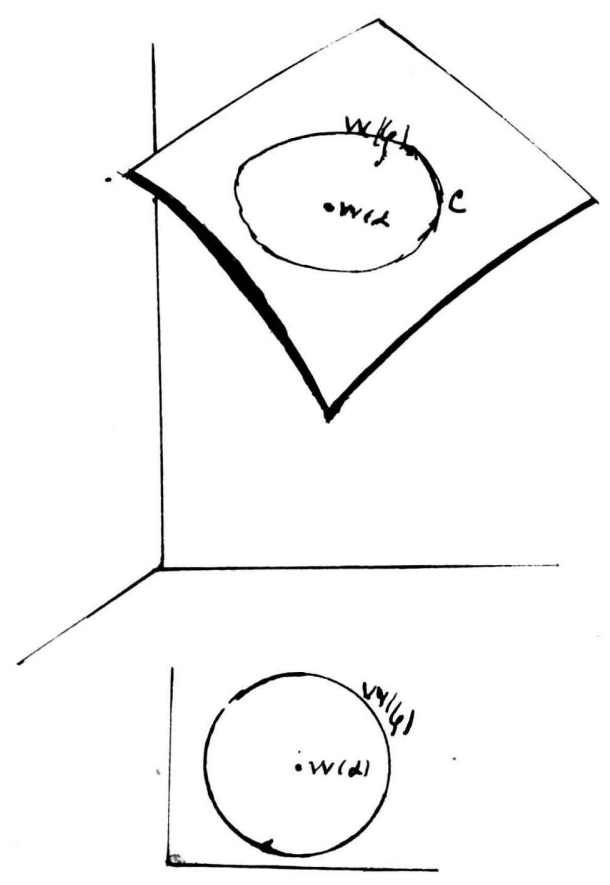

Fig. X.

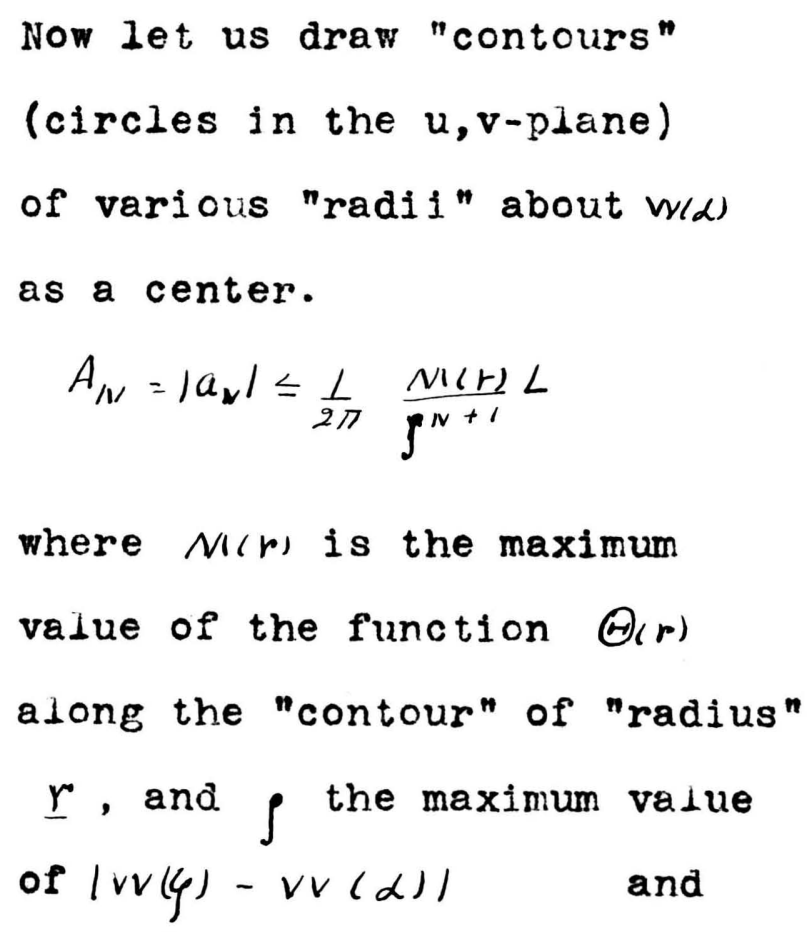

thesis $\Theta(r)$ is always less than $/ T$, hence

$$
\frac{L M(r)}{\rho^{N+1}} \leqq \frac{r \dot{L}}{\rho^{N+1}}
$$

Therefore by increasing $\rho, u_{N}$ can be made as small as we please. But $a_{v}$ is a definite integral, therefore, if $v \geqslant 1, a_{N}=0$. Hence $(r)$ is a constant $a_{0}$. Liouville's theorem may also be stated as follows:

A Punction $\Theta(r)$ which is regutar everywhere, including the point at infinity, is a constant.

9. The singularities of single-valued functions.

Let us consider a function $\Theta(r)$ which is regular at all points in the region $A$ of the surface $\sum$, except at 

the point $V(\alpha)$, so that the point $N V(\alpha)$ is a singularity

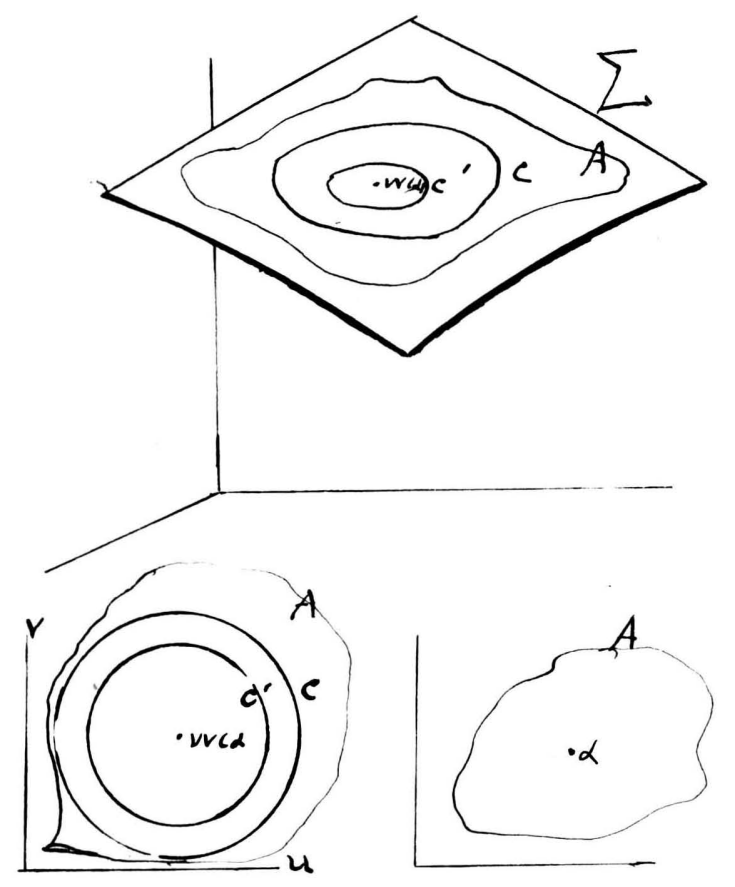

Fig. XI . of the function $\Theta(r)$. Let us draw a "contour" $C^{\prime}$ about $v v(\alpha)$ as center with a "radius" $r$. Then the function $\Theta(r)$ is regular in the region bounded by the two "contours" $C$ and $C^{\prime}$, where $C$ is a "contour" with a larger "radius"; and the Laurent expansion holds, Hence

$$
\begin{aligned}
\Theta(r)= & \left.J_{0}+J_{1} 2 v v(r)-v v(\alpha)\right]+J_{2}[v v(r)-v v(\alpha)]^{2}+ \\
& \cdots+\frac{/ r_{1}}{v v(r-v v(\alpha)}+\frac{K_{2}}{[v v(r)-v v(\alpha)]^{2}}+-
\end{aligned}
$$

The terms in the last line of this expansion are those whichtell us of the nature of the singularity of $v v(\alpha)$. It is evident that if none of these terms exist, that there is no singularity at the point.

If the number of terms of the last line is unlimited, that is, if the terms with the $/ K^{\prime}$ far coefficients form an infinite series, the point $\alpha$ in the $p, q$-plane, or the corresponding point $V V(\alpha)$ on the surface $\sum$ is said to be an essentially singular point for the function $\Theta(r)$. 

On the other hand, if these terms are finite in number, and if at least one of these exists, that is, if all the $/ K^{\prime} s$ are not zero, the point $\alpha$ in the $p, q$-plane, or the corresponding point $w(\alpha)$ on the surface, is said to be a pote. If all the $K^{\prime} s$, except $K_{1}$, equal zero, the point $v v(\alpha)$ is said to be a simple pole. If $K_{N}$ is the highest $K$ which does not equal zero, $w(\alpha)$ is said to be a pole of order n. If the singularity is a pale of order $n$, the function $[v v(r)-v v(\alpha)]^{N} \Theta(r)$ is regular throughout the region $A$.

The function $e^{\frac{1}{w r}}$ has an essentially singular point at $w(r), r i o$. In order to see this, let us recall the expansion of $e^{x}$.

$$
e^{x}=1+x+\frac{1}{2 !} x^{2}+\ldots
$$

from this we see that

$$
e^{\frac{1}{w(r)}}=1+\frac{1}{n /(r)}+\frac{1}{2 !}\left[\frac{1}{v v(r)]^{2}+\cdots}-\frac{1}{\frac{\pi}{N !}[w(r)]^{N}+\cdots}\right.
$$

10. Functions with no essential singular point. Theorem: If a single-valued function $\Theta(r)$ has no singularities, other than poles, over the entire surface, inciuding the point at infinity, the function is a rational function in $\underline{v(r)}$.

Let us suppose that the poles are at the points $\alpha_{1}, \alpha_{2} \ldots \alpha_{l}$, in the $p, q-p l a n e$, or at the corresponding 

points $v v\left(\alpha_{1}\right), v v\left(\alpha_{2}\right), \ldots w\left(\alpha_{2}\right)$ on the surface, then the Laurent expansion at the polew $\left(\alpha_{i}\right)$, where $N_{i}$ is the order of the pole, is

$$
\frac{K_{i 1}}{w(r)-w\left(\alpha_{i}\right)}+\frac{Y_{i 2}}{\left[w(r)-w\left(\alpha_{i}\right)\right]^{2}}+\cdots+\cdots+\frac{1 Y_{i N_{i}}}{\left.2 W(r)-v v\left(\alpha_{i}\right)\right]^{N_{i}}}
$$

If the point $r=\infty$ be a pole of order $N$, the Laurent expansion will be

$$
\alpha_{1} w(r)+\alpha_{2} L v(r) J^{2}+\ldots \ldots+\alpha_{N}\left[v(r) J^{N} .\right.
$$

The function

$$
\begin{aligned}
\Theta(r) & -\sum_{i=1}^{i=\rho} \frac{r_{i}}{v v(r)-v v\left(\alpha_{i}\right)}+\frac{r_{i 2}}{\left.L v(r)-v v\left(\alpha_{i}\right)\right]^{2}}+\cdots+\frac{r_{i w_{i}}}{\left[v v(r)-w\left(\alpha_{i}\right)\right]^{N}} \\
& -\left[d_{1} v v(r)+d_{2}[w(r)]^{2}+\ldots+d_{r}[w(r)]^{N}\right]
\end{aligned}
$$

since it is regular over the entire surface is, by Liouvilie's theorem, equal to sme constant $a_{0}$. Therefore, the function $\Theta(r)$ may be written

$$
\begin{aligned}
& \Theta(r)=a_{0}+a_{1} r v(r)+a_{2}[r(r)]^{2}+\ldots \ldots+d_{N}[v V(r)]^{N} \\
& +\sum_{i=1}^{i=l} \frac{K_{i 1}}{w(r)-w v\left(\alpha_{i}\right)}+\frac{K_{i \cdot 2}}{\left[w(r)-w\left(\alpha_{i}\right)\right]^{2}}-\cdots+\frac{K_{i} v_{i}}{\left[w w(r)-w\left(\alpha_{i}\right)\right]^{N_{i}}}
\end{aligned}
$$

and hence, since $\Theta(r)$ is expressed as a rational function in $v(r)$, the theorem is proved.

11. Corollary: A function which is single-valued and has no singularities over the entire surface, other than a pole at the point at infinity, is a potynomial in $v v(r) \cdot$ 

If the only singularity is a pole at infinity, all the $K^{\prime} g$ in the above expansion of $\Theta(r)$ equal zero, therefore

$$
\Theta(r)=a_{0}+u_{1} w(r)+a_{2}[v v(r)]^{2}+\cdots
$$

and hence the corollary is proved.

\section{Logarithmic Residues.}

Let $w(\alpha)$ be a pole of order $m$, or an isolated essential singular point of the function $\theta(r)$, and

$w(r)$ a regular function within the neighborhood of the point $V(\alpha)$. The Laurent expansion of the function

$\theta(r)$ about the point $W(\alpha)$ is

$$
\frac{K_{M}}{[v v(r)-w(\alpha)]^{\mu}}+\frac{K_{M-1}}{2 v v(r)-v v(\alpha)]^{M-1}}-\cdots+\frac{K_{1}}{w(r)-w(\alpha)}+\psi(r)
$$

where $\psi(r)$ is regular within the neighborhood of the point $w(\alpha) \quad$.

$$
\text { Let } C^{\prime} \text { be a "contour" with center at vr(d) on }
$$

the surface $\sum$. Then we have

$$
\int_{c^{\prime}} \Theta(r) d w(r)=\int_{e^{\prime}} \sum_{j=1}^{j=m} \frac{k j d v(r)}{2 v(v)-v v(\alpha) j j}+\int_{c^{\prime}} \psi(r) d w(r)
$$

Now $\int_{e} \phi(r) \alpha w(r)=0$, since $\psi(r)$ is regular within the "contour" $C$ ' and the Cauchy integral formula applies, and $\int_{e} \frac{d w(r)}{\left[v(r)-w(H) j^{\prime}\right.}=0$ except when $j=1$, because the function $\frac{1}{2 w(r)-w(\alpha) j^{j-1}}$ takes on the same value after it has 

described a closed path. On the other hand, $\int_{C^{\prime}} \frac{\alpha_{w(r)}}{w(r)-v v(\alpha)}=2 \pi i$ as was proved in Sec. II., 4.

Therefore,

$$
\int_{e^{\prime}} \Theta(r) d w(r)=/ r_{i} 2 \pi i
$$

The coefficient $K_{i}$ is called the residue of the function $\Theta(r)$ with respect to the singular point $w\left(\alpha_{i}\right)$. Let us consider now a closed contour $\Gamma$, which has in its interior a finite number of singular points $a, b, c . . \ell$. Let

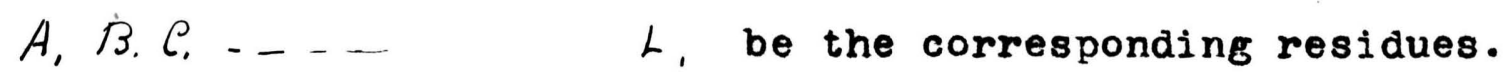
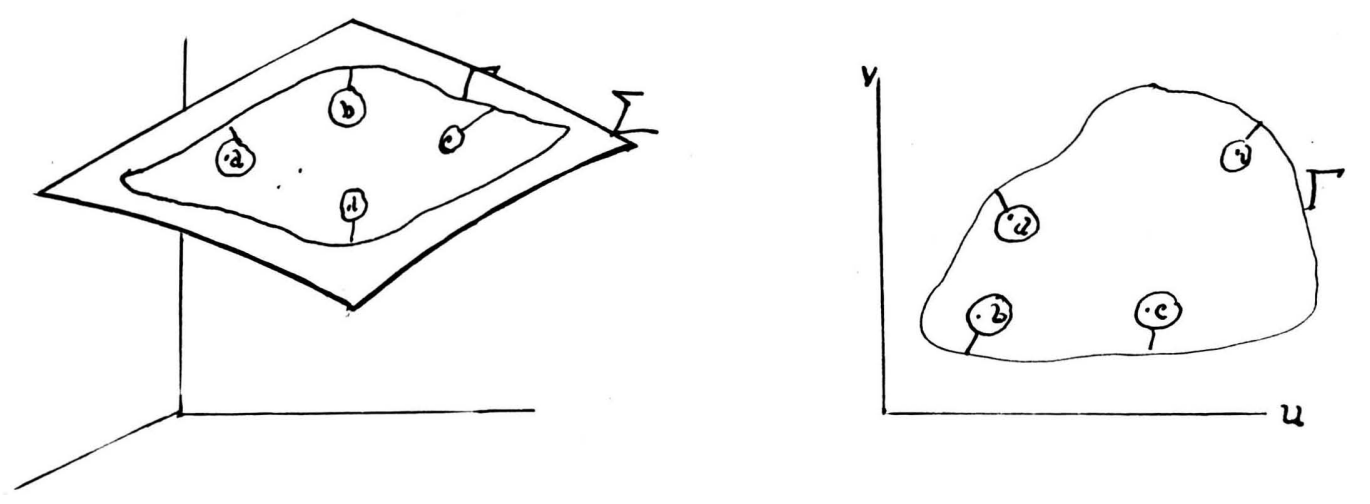

FIg. XII.

If we surround each of these points by "contours" (circles in the $u, v$-plane) with very small "radii", the integrad taken around $\Gamma$ is equal to the integral taken around the small "contours", their sense being the same, hence we have

$$
\int_{r} \theta(r) d w(r)=2 \pi i(A+B+C+\cdots+L)
$$

which we may state as follows: 

56.

The integral $\int \Theta(r) d v(r)$ taken along $\Gamma$ in the direct sense, is equal to the product of $2 \pi i$ by the sum of the residues relative to the singular points of

enclosed by the closed path

13. Multiple-valued functions. - Branch points.

In all our previous work, we have supposed that all the functions that we considered were single-valued functions of $r$; however, there, as in the case of analytic functions, there may exist functions which are many-valued functions of

$r$, i.e., which have more than one value corresponding to each value of $r$.

The points and curves for which the vaiues of the functions of different branches coincide are called branch points and branch curves.

In the case of anaiytic functions, we found that there were no branch curves, but only branch points. Let us investigate the classes of functions that wo are studying and see if it is possible to have branch curves.

For these branch points or branch curves, the Jacobian of transformation(i) vanishes - hence, if we have the function $w(r)=u(p, q)+v(p, q) i$, at the branch point, or branch curves, the determinant

$$
\left|\begin{array}{ll}
\frac{\partial u}{\partial p}, & \frac{\partial u}{\partial q} \\
\frac{\partial v}{\partial p} & \frac{\partial v}{\partial q}
\end{array}\right|
$$

(1) Cf. Goursat; op. cit., vol. I., p. 52. 

vanishes.

$$
\text { If we replace } \frac{\partial v}{\partial p} \text { and } \frac{\partial v}{\partial q} \text { by their values obtained }
$$

from the Beltrami equations, we have the quadratic form

$$
\frac{E}{H^{\prime}}\left(\frac{\partial u}{\partial q}\right)^{2}-\frac{2 F}{H} \frac{\partial u}{\partial p} \frac{\partial u}{\partial q}+\frac{G}{H}\left(\frac{\partial u}{\partial p}\right)^{2}
$$

which is always definite unless

$$
\frac{\partial u}{\partial p}=\frac{\partial u}{\partial q}=0
$$

In the same way by replacing in the determinant the values of $\frac{\partial u}{\partial p}$ and $\frac{\partial u}{\partial q}$ obtained from the Beltrami equations, we have the quadratic form

$$
\frac{E}{H}\left(\frac{\partial r}{\partial q}\right)^{2}-\frac{2 F}{H} \frac{\partial v}{\partial p} \frac{\partial \alpha}{\partial q}+\frac{G}{H}\left(\frac{\partial v}{\partial p}\right)^{2}
$$

which is always definite unsess

$$
\frac{\partial v}{\partial \rho}=\frac{\partial v}{\partial q}=0
$$

Hence the condition that the determinant vanish is that

$$
\frac{\partial u}{\partial p}=\frac{\partial u}{\partial g}=\frac{\partial v}{\partial p}=\frac{\partial v}{\partial q}=0
$$

which expresses the condition that there be oniy branch points, and not branch curves, therefore, in general, we have branch points.

14. Example of many-valued functions.

Let us consider the well known transformation

$$
\begin{aligned}
& u=p \cos q \\
& y=p \sin q .
\end{aligned}
$$

(i) cf. Darboux: op. cit., voI. III, p. 194. 

58.

The function $W=u+v i$ is a non-analytic

function. It, however, is a function which belongs to

the classes we have defined, and we are in a position to

study it. The function $V=2 l+v i$ belongs to the sur-

face $\sum$ whose linear element is defined by

$$
d s^{2}=\frac{1}{p} d p+p d q^{2}
$$

which is mapped upon the $u, v-p$ lane by a conformal transformation, hence we can study the above transformation best by drawing the Riemann surfaces for the transformation of the $p, q-p l a n e$ upon the $u, v$-plane.
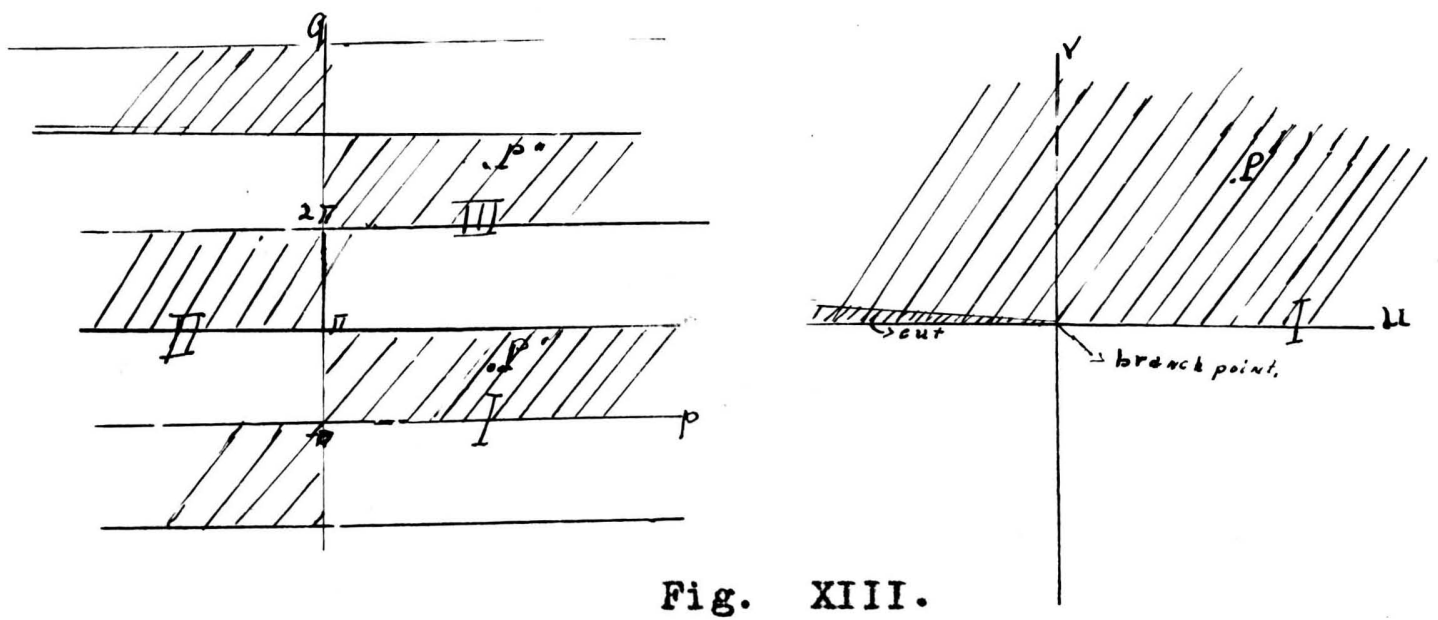

Fig. XIII.

The Jacobian of transformation is $p$; therefore when we have the branch point, or branch curves; in the $u, v-$ plane it is a point, namely, the origin - in the p,qplane a line, namely, the q-axis.

There will be an infinite number of sheets in the $u, v-p l a n e$, corresponding to the one sheet over the 

p,q-plane. Corresponding to any geometrical point $P_{0}$ in the shaded part of the $u, v-p l a n e$ there will be an infinite number of geometrical points in the $p, q$-plane, $P^{\prime}, P^{\prime \prime}, \rho^{\prime \prime}, \ldots$ one in the shaded portion of each of the strips of width $2 \pi$. The infinite number of sheets over the $u, v-p l a n e$ will hang together at the origin.

$$
\text { If we consider the function } W^{\prime}=w^{2} \text {, we know }
$$

that, since $W^{\prime}$ is an ordinary analytic function of $V /$, it belongs to the same surface $\Sigma$ to which $w$ belongs. The function $W^{\prime}=2 L^{\prime}+v^{\prime} i=V v^{2}$

where

$$
\begin{aligned}
& u^{\prime}=p^{2} \cos 2 q \\
& v^{\prime}=p^{2} \sin 2 q .
\end{aligned}
$$

The Riemann surface for these functions wi $i d$ be just as the above, except that the strips will be of width $\pi$, and there will be a compression in toward the $q$-axis, as indicated in the figure.
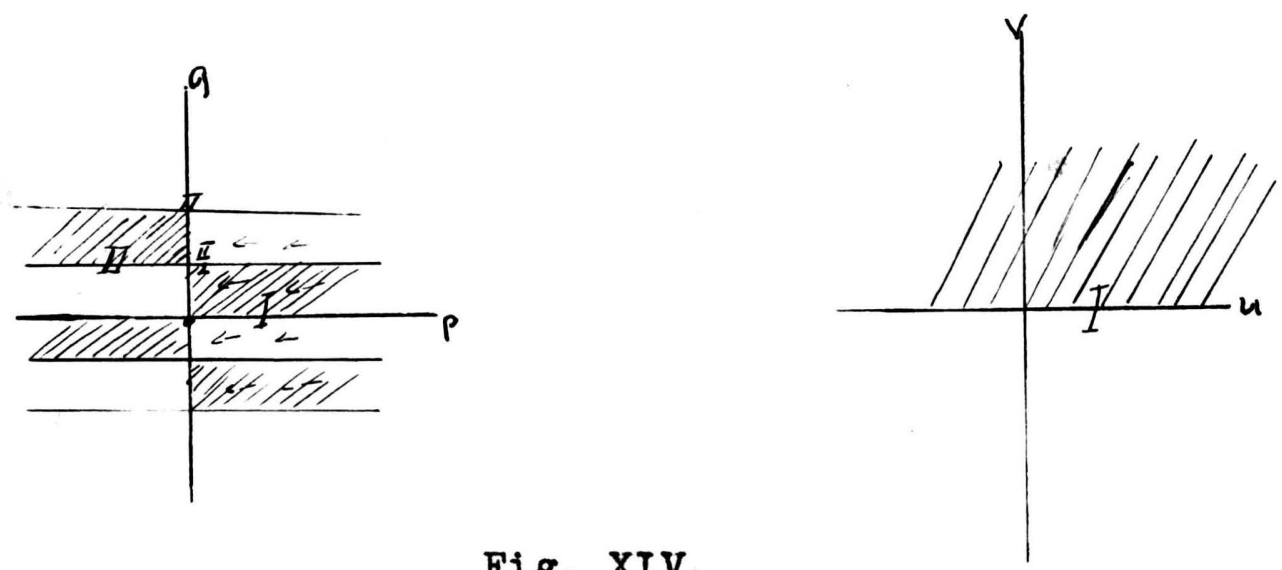

Fig. XIV.

15. Region of Convergence.

In the foregoing proof of the Cauchy-Taylor and 

60.

Laurent series, we made no statement about the maximum size of the region of convergence. If we are thinking of the function as a function in the $u, v-p l a n e$ we may speak of the circle of convergence, which will as in thecase of analytic functions bend until it touches some singularity or branch point. On the other hand, if we are thinking of the function as a function on the surface $\sum$ or on the $p, q$-plane, the region of convergence is no longer a circle, but some "contour" which corresponds to the circie on the $u, v$-plane. This "contour" will extend until it touches some singuiar1ty; and it will shrink up on the point $w(\alpha)$ as the corre-

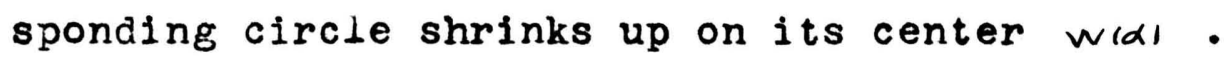



61.

Section IV.

1. Examples of some of these functions.

As we have before stated, these functions which

we are studying are mereiy the solutions of the Beltrami

diferential equations

$$
\begin{aligned}
& \frac{\partial v}{\partial p}=\frac{E \frac{\partial u}{\partial q}-F \frac{\partial u}{\partial p}}{H} . \\
& \frac{\partial v}{\partial q}=\frac{F \frac{\partial u}{\partial q}-G \frac{\partial u}{\partial p}}{H} .
\end{aligned}
$$

In order to find some function satisfying these differential equations let us take definite surfaces.

a) Let us consider the sphere which is defined by the equations,

$$
\begin{aligned}
& x=R \sin p \cos q \\
& y=R \sin p \sin q \\
& z=R \cos p
\end{aligned}
$$

then the square of the linear element on the sphere is given by the equation

$$
d s^{2}=R^{2} d_{p}^{2}+R^{2} \sin ^{2} p d_{y}^{2} \text {. }
$$

Hence, in this case, the Beltrami equations become 



$$
\begin{aligned}
& \frac{\partial v}{\partial p}=\frac{R^{2} \frac{\partial u}{\partial q}}{R^{2} \sin p} \\
& \frac{\partial v}{\partial q}=-\frac{R^{2} \sin ^{2} p \frac{\partial u}{\partial p}}{R^{2} \sin p}
\end{aligned}
$$

Now we wish to find one particular solution of these equations. Let us assume that $v$ is a function of $p$ alone, and that $u$ is a function $q$ alone, then the second of the equations is satisfied and the first becomes

$$
\sin p \frac{d v}{d p}=\frac{d u}{d q}
$$

of which $v=\log \frac{p}{2}, u=q$ is a solution.

Therefore the function $w=u+v i=q+i \log \frac{p}{2} \quad$ is one of the functions that we are seeking. Any ordinary analytic function of $w$ is also a function of this class. (i)

b) The linear element of the pseudo-sphere is given by the equation

$$
d s^{2}=d^{2}\left(d p^{2}+e^{x p} \alpha^{2} q\right) \text {. }
$$

where the pseudo-sphere is generated by the revolution of the tractrix,

$$
p=a \sin \phi, \quad z=\log \left(\tan \frac{\varphi}{2}+\cos \varphi\right)
$$

about its base! (1i)

In this case

$$
E=d^{2}, \quad F=0, G=d^{2} e^{2 p} \text {. }
$$

(i) See E. Picard: op. cit., vol. II., p. 541-2.

(i1) Darboux: op. cit., vol. III., p. 394. 

Hence the Beltrami equations become

$$
\begin{aligned}
& \frac{\partial v}{\partial p}=\frac{a^{2} \frac{\partial u}{\partial q}}{a^{2} e^{p}} \\
& \frac{\partial r}{\partial q}=\frac{-a^{2} e^{2 p} \frac{\partial u}{\partial p}}{a^{2} e^{p}} .
\end{aligned}
$$

Let us as in the foregoing example assume that $V$ is a function of $\beta$ alone, and $u$ of $q$ alone, then we find that the above equations will be satisfied by

$$
v=-e^{-p}, u=q
$$

Therefore any analytic function of $\quad W=2 l+v i$ is a function of the second kind.

c) Let us now take the torus which is given by the equations

$$
\begin{aligned}
& x=(R-\rho \cos p) \cos q \\
& y=(R-\rho \cos p) \sin q \\
& z=\rho \sin p
\end{aligned}
$$

where $\rho$ is the radius of the generating circte and $\mathbb{P}$ the distance of its center from the axis.

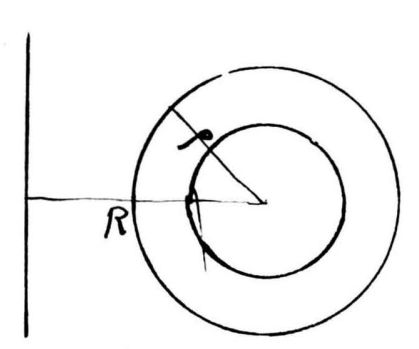

The linear element of the surface is defined by the equation

$$
\begin{aligned}
& d s^{2}=\rho^{2} d p^{2}+(\beta-\rho \cos \rho)^{2} d{ }^{2} \\
& \text { Hence, } E=\rho^{2}, F=0, G=(p-\rho \cos q)^{2}
\end{aligned}
$$

therefore the BeItrami equations become 



$$
\begin{aligned}
& \frac{\partial v}{\partial p}=\frac{\rho^{2} \frac{\partial u}{\partial q}}{\rho(R-\rho \cos \phi)} \\
& \frac{\partial v}{\partial q}=\frac{-(R-\rho \cos p)^{2} \frac{\partial u}{\partial p}}{\rho(R-p \cos p)}
\end{aligned}
$$

If we assume again that $L$ is a function of $p$ alone, and $v$ of of alone, we see that one solution of the above is

$$
u=\int_{0}^{p} \frac{\rho d p}{R-\rho \cos p}, \quad v=q
$$

Here we have as one solution $v y=u+v i$, a function which is uniform upon the torus and which is a doubly-periodic function with the periods $2 \pi$ and

$$
\int_{0}^{2 \pi} \frac{p d p}{p-\rho \cos p}
$$

The above examples of functions of these classes are the well-known functions which define a conformal transformation of the particular surface to which each belongs upon a plane. But, while it is evident by the restriction we have placed upon $u$ and $v$, that all the functions which belong to the classes that we have defined, will give a conformal transformation of some surface upon the plane, we have here reached our functions in a way very different from the way in which these functions are ordinarily reached, namely in seeking a solution for the Beltrami equations.

(i) cf. E. Plcard: op. cit., vol. II., p. 544. For Riemann surfaces of this function see Klein: On Riemann's Theory of Functions, p. 46. 

And we can obtain functions that belong to this class by finding merely the solutions of the Beltrami equations, where $E, F$, and $G$ are any arbitrary functions of $p$ and $q$.

2. Interpretation of this work in the $u, v$-plane.

The surface $\sum$ which plays such an important part in all this work is not essential to theory, but is rather an aid to our intuition and a help in the anticipation of results. The $u, v-p l a n e$ is essentially the plane in which we are working. In order to interpret this work in the $u, v-p l a n e$, in the case of differentiation and integration it is necessary merely to make new definitions - namely, by derivative we shaIl mean $\frac{d v v^{\prime}}{d v v}$, and by the integral of $\Theta(r)$ from a to $\underline{b}$, we shall mean $\int_{d l}^{b} \Theta\left(H\left(\eta+\varphi_{i}\right)\left(\sqrt{E} d_{p}+\frac{F+i H}{\sqrt{E}} d q\right)\right.$ However, if we attempt to interpret the isogonal transformation, we are forced into a ncn-Euclidean geometry in the u,v-plane, A geometry about which very little can be said until we know the surface to which the functions belong, or the $E, F$, and $G$ which occur in the Beltrami equations. In all cases the geometry in the $u, v$-plane will be one in which extremals are perpendicuiar to transversals! (i)

(i) Stromquist: On geometries in which circles are the the shortest 1 ines. Transactions of the American Mathematical Society, vol. II., No. 2 , p. 181. 

66.

If we take a particular surface, we can make further statements about the geometry. In the case of the pseudosphere, which we gave above $(1, b)$ the geometry is Lobatchewskian, that is, a geometry in which circles are the shortest distances and extremals are perpendicular to their transversals. (i)

(1) Stromquist: op. cit. 



\section{Conclusion}

It is interesting to note some of the problems in which the Beltrami equations occur. In a brief statement of a fev of these problems, it is impossible to give a treatment comprehensive and rigorous enough to give a clear idea of what has been done; therefore, the attempt here will be to indicate merely the general lines of the work.

Professor Plcard obtains these equations by equating one of the conjugate factors of $d_{i 2}^{2}+d r^{2}$ to the corresponding factor of $\lambda\left(E d \frac{t}{p}+2 F d p d q+\sigma d \frac{2}{q}\right)$, and in his chapter on Theoremes Generaux sur une Surface de Riemann (i) he proves a few theorems about functions that satisfy the Beltrami equations. However, he turns from the consideration of these functions with little more than the suggestion that, since any function belonging to a surface is an ordinary analytic function of any other function of this same surface, all functions of a surface may be treated as analytic functions of some one function of this surface.

Professor Darboux, following Beltrami, proves several very interesting theorems about these functions in his work on the surface theory. Among these is a theorem for

(1) Picard: op. cit., vol. II, p. 541 . 

68.

the surface analogous to the Green theorem for the plane. (i) Professor Klein in his work on Riemann's Theory of Functions interpretes these functions that satisfy the Beltrami equations of the second order as steady streaming along the surfece. In this very interesting work he gives examples of the stream lines for several different surfaces. His treatment is from the geometrical rather than the functional standpoint.

In speaking of an extension of the theory of functions of a complex variable to include non-analytic functions, we said that non-analytic functions need not be necessarily useless or difficult; and the functions which this extension has lead us to consider, as is obvious from the exarnples given in the preceding paragraph, are not useless and difficult, but the well-known functions which arise in the theory of surfaces. While the extension of the theory of functions of a complex variable that we have made is not the most generat one - namedy, one in which we will not be forced to consider the functions belonging to each different surface as forming a separate class, and in which, even when thus considered, we will not have to make such frequent use of a

(i) Darboux: op. cit., vol. III., p. 198, et sq. 

69.

standard function of that class, - yet it does enable us to make a more thorough and accurate study of the functions than we could otherwise. As an illustration of this take the method indicated by Professor Picard ( op. cit., vol. II, p. 541). His definition of a pole is essentially the same as the one we adopted, and yet in following out his suggestions ( Loc. cit.), we should scarcely be lead to consider whether the standard function $V(N)$ (of which we are to consider all of functions $\Theta(r)$ as an ordinary analytic function) is double-valued or not. Moreover, this extension will lead us to many functions which wouid not be encountered in the surface theory, because we are in the position to study any functions which are the solutions of the Beltrami equations,

$$
\begin{aligned}
& \frac{\partial u}{\partial q}=F \frac{\partial u}{\partial p}-H \frac{\partial v}{\partial p} \\
& \frac{\partial v}{\partial q}=F \frac{\partial v}{\partial p}+H \frac{\partial u}{\partial p} .
\end{aligned}
$$

where $H=\sqrt{E G-F^{2}}$, and where $E, F$, and $G$ are any arbitrary functions of $p$ and $q$, and it is not necessary to know anything about the surface other than the values of $E, F$, and G. 

Burkhardt, Heinrich. Funktionentheorishe Vorlesungen.

Cauchy, Augustin. Sur les fonctions de variables imaginaires. Comptes Rendus: $1851,1^{\text {er }}$ sem., p. 160.

Sur L'application de 1 a nouvelle theorie

des imaginaires aux diverses branches des

sciences matheratigues.

Comptes Rendus: $1847: 1847,2^{e}$ ser., p. 129.

Darboux, Gaston. Lecons sur Ia Theorle Generale des Surfaces. Gauthier-Villiers et Fils, Paris, 1894.

Durege, H. Elements of the Theory of Functions.

(Translated from the German by G. E. Fisher and I. J. Schwatt.)

J. S. Cushing \& Company, Norwood, Mass. 1896.

Forsyth, A. R. Theory of Functions of a complex Variable.

The University Press, Cambridge. 1900.

Goursat, Edouard. Cours d'Analyse Mathematique. Vol. II.

Gautier-Villars, Paris. 1905.

Harkness and Morley. Introduction to Anajytic Functions.

Macmillan \& Company, London. 1898.

Harnack, Alex. Introduction to Calculus.

(Translated from the German by George $L$. Cathcart.)

Williams \& Norgate, Convent Garden, London. 1891.

Hobson, E. W. The Theory of Functions of a Real Variable.

The University Press, Cambridge. 1907.

Humbert, G. Cours d'Anajyse. Vols. i, 2.

Gauthier-Villars, Paris. 1882. 



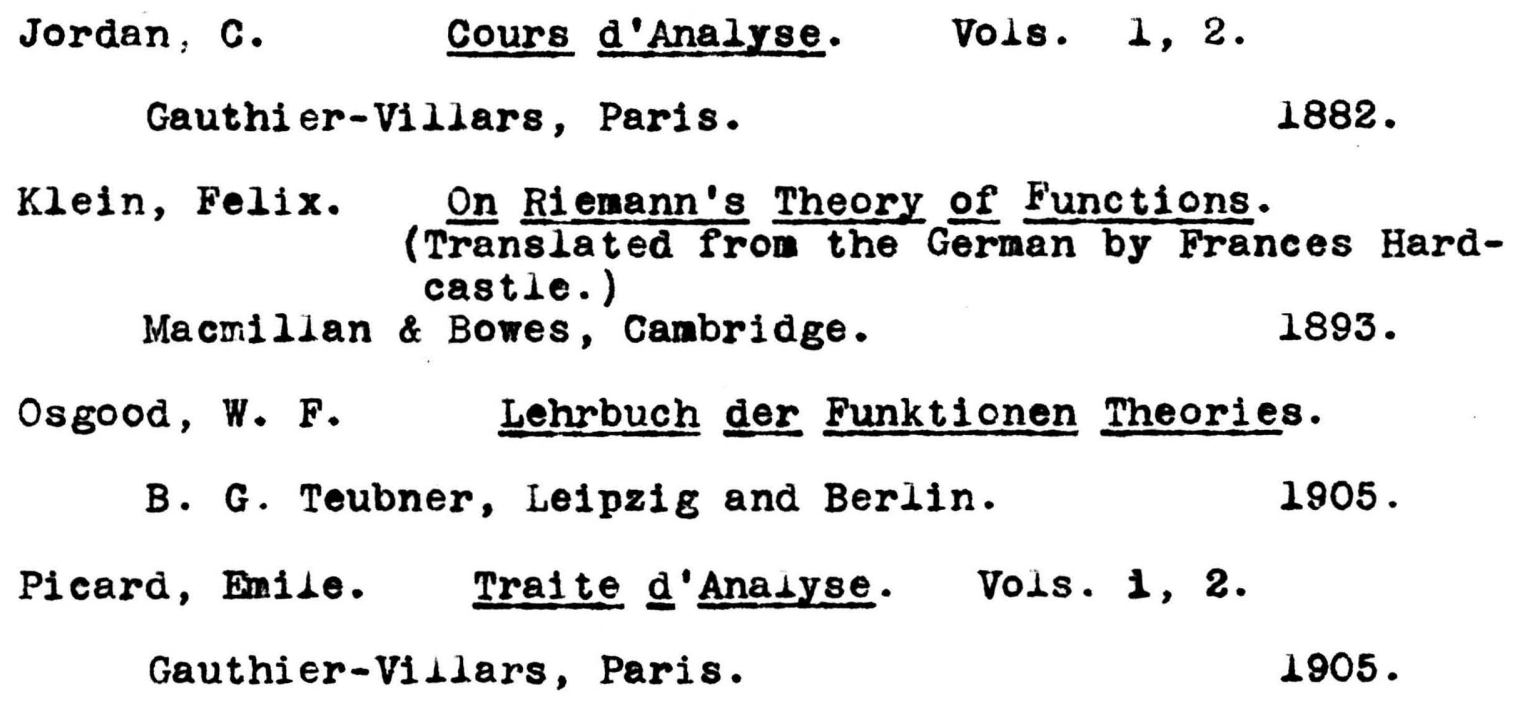

Pringsheim, Alfred. Grundlagen der Allgemeinen Funktiontehre.

Encyclopadie der Mathematischen Wissenschaften. II.A 1

Stromquist, Cart Eben. On Geometries in which circles are the shortest 1ines.

Transactions of the American Mathematical Society. Vol. 7, No. 2, pp. 175 - 183. April, 1906.

Thomae, J. Elementare Theorie den analytischen Funktionen.

Halle, a. s., Verlag von Louis Nebart. 1898.

Valson, C. A. La Vie et les Travaux du Baron Cauchy.

Gauthier-Villars, Paris. 1868.

Whit taker, E. T. A Course of Modern Analysis.

The University Press, Cambridge. 1902. 







\section{$378.7 M 71$ \\ $X W 41$}

Neither is it $\%$ o be chresed out overnight. 
